Aritoshi Iida $\cdot$ Susumu Saito $\cdot$ Akihiro Sekine

Takuya Kitamoto $\cdot$ Yuri Kitamura $\cdot$ Chihiro Mishima

Saori Osawa · Kimie Kondo • Satoko Harigae

Yusuke Nakamura

\title{
Catalog of 434 single-nucleotide polymorphisms (SNPs) in genes of the alcohol dehydrogenase, glutathione S-transferase, and nicotinamide adenine dinucleotide, reduced (NADH) ubiquinone oxidoreductase families
}

Received: March 15, 2001 / Accepted: April 6, 2001

\begin{abstract}
An approach based on development of a large archive of single-nucleotide polymorphisms (SNPs) throughout the human genome is expected to facilitate large-scale studies to identify genes associated with drug efficacy and side effects, or susceptibility to common diseases. We have already described collections of SNPs present among various genes encoding drug-metabolizing enzymes. Here we report SNPs for such enzymes at additional loci, including 8 alcohol dehydrogenases, 12 glutathione S-transferases, and 18 belonging to the NADHubiquinone oxidoreductase family. Among DNA samples from 48 Japanese volunteers, we identified a total of 434 SNPs at these 38 loci: 27 within coding elements, 52 in $5^{\prime}$ flanking regions, five in $5^{\prime}$ untranslated regions, 293 in introns, 20 in $3^{\prime}$ untranslated regions, and 37 in $3^{\prime}$ flanking regions. The ratio of transitions to transversions was approximately 2.1 to 1 . Among the 27 coding SNPs, 13 were nonsynonymous changes that resulted in amino acid substitutions. Our collection of SNPs derived from this study should prove useful for investigations designed to detect associations between genetic variations and common diseases or responsiveness to drug therapy.
\end{abstract}

Key words Single-nucleotide polymorphism $(\mathrm{SNP}) \cdot$ Alcohol dehydrogenase - Glutathione S-transferase - NADH ubiquinone oxidoreductase . High-dense SNP map · Nonsynonymous substitution $\cdot$ Japanese population

A. Iida $\cdot$ S. Saito $\cdot$ A. Sekine $\cdot$ T. Kitamoto $\cdot$ Y. Kitamura C. Mishima $\cdot$ S. Osawa $\cdot$ K. Kondo $\cdot$ S. Harigae $\cdot$ Y. Nakamura Laboratory for Genotyping, The SNP Research Center, Institute of Physical and Chemical Research (RIKEN), Tokyo, Japan

Y. Nakamura $(\bowtie)$

Laboratory of Molecular Medicine, Human Genome Center,

Institute of Medical Science, The University of Tokyo, 4-6-1

Shirokanedai, Minato-ku, Tokyo 108-8639, Japan

Tel. +81-3-5449-5372; Fax +81-3-5449-5433

e-mail: yusuke@ims.u-tokyo.ac.jp

\section{Introduction}

Human genetic variations result from a dynamic process over time that includes sudden mutations, random genetic drift, and, in some cases, founder effects. Variations at a single gene locus are useful as markers of individual risk for adverse drug reactions or susceptibility to complex diseases (for reviews, see Risch and Merikangas 1996; Kruglyak 1997; McCarthy and Hilfiker 2000). Common types of sequence variation in the human genome include singlenucleotide polymorphisms (SNPs), insertion/deletion polymorphisms, and variations in the number of repeats of certain motifs (e.g., microsatellites and variable number of tandem repeat loci). Among those variations, SNPs are the most abundant, stable, and widely distributed across the genome; moreover, they lend themselves to automated analysis on a very large scale with high-throughput typing technologies (for reviews, see Wang et al. 1998; Halushka et al. 1999).

Projects to establish large collections of SNPs at candidate loci and to develop detailed SNP maps across the human genome have been under way for some time in various laboratories (Sherry et al. 2000, 2001). Construction of an SNP database for the Japanese population began in 2000, and already around 34,000 SNPs have been archived (http://snps.ims.u-tokyo.ac.jp). Simultaneously, we have been focusing on identifying SNPs within genes encoding drug-metabolizing enzymes, drug receptors, and transporters. Local, highly dense SNP maps have been completed for four gene families related to drug metabolism, including 17 members of the S-transferase family, three quinone oxidoreductases, two epoxide hydrolases, and five $\mathrm{N}$-acetyltransferases (Iida et al. 2001; Saito et al. 2001; Sekine et al. 2001). Such genes are likely to have significant roles in responsiveness to drugs and/or in susceptibility to common diseases such as arteriosclerosis, diabetes mellitus, and cancer.

In this study we report a total of 434 SNPs that we identified in a Japanese population sample by direct sequencing of 38 gene loci encoding alcohol 
dehydrogenases, glutathione S-transferases, and nicotinamide adenine dinucleotide, reduced (NADH) uniquinone oxidoreductases.

\section{Subjects and methods}

DNA samples and genes encoding drug-metabolizing enzymes

Blood samples were obtained, with informed consent, from 48 healthy Japanese individuals for this study, which was approved by the SNP Research Center, RIKEN. Genomic DNA was extracted from each sample according to standard protocols. On the basis of DNA sequences released from the GenBank database, we predicted structures and designed primers to amplify each gene in its entirety as well as up to $2 \mathrm{~kb}$ upstream from the first exon (putative promoter region) and downstream from the last exon. Accession numbers are shown at the top of each gene map in Fig. 1. Regions corresponding to repetitive sequences were predicted by the RepeatMasker program with the option "Do not mask simple repeats and lowcomplexity DNA" selected (http://ftp.genome.washington. edu/cgibin/RepeatMasker).

Polymerase chain reaction (PCR), direct sequencing, and detection of SNPs

PCR experiments and DNA sequencing were performed according to methods described previously (Ohnishi et al. 2000). Each PCR experiment was carried out using $20 \mathrm{ng}$ of DNA pooled from three individuals. All SNPs detected by the PolyPhred computer program (Nickerson et al. 1997) were confirmed by sequencing both strands of each PCR product.

\section{Results and discussion}

We present here a series of highly dense SNP maps that include a total of 434 SNPs among 38 genes belonging to three families of enzymes associated with drug metabolism. The genomic structures and locations of identified SNPs are illustrated schematically in Fig. 1. Eight of the genes studied here belong to the alcohol dehydrogenase family, 12 to the glutathione S-transferase family, and 18 to the NADH ubiquinone oxidoreductase family. Of the SNPs identified, $334(77 \%)$ were not previously included in the National Center for Biotechnology Information (U.S.) dbSNP database.

\section{Alcohol dehydrogenase family}

The family of alcohol dehydrogenases (EC1.1.1.1) in vertebrates consists of several enzymes that are able to catalyze reversible oxidation of a wide variety of endogenous and xenobiotic primary and secondary alcohols, to produce the corresponding aldehydes and ketones. Several distinct classes of vertebrate alcohol dehydrogenase, encoded by nonorthologous genes, have been defined on the basis of sequence homologies and/or unique catalytic properties or gene expression patterns (for reviews, see Duester et al. 1999; Ashmarin et al. 2000). We analyzed here six of the seven known $A D H$ gene loci and two genes that had shown sequence similarities to $A D H$ by direct sequencing (Fig. 1, a1-a8). A total of 66 SNPs were identified from these eight loci: eight were located in coding elements, eight in $5^{\prime}$ flanking regions, one in a $5^{\prime}$ untranslated region, 37 in introns, eight in $3^{\prime}$ untranslated regions, and four in $3^{\prime}$ flanking regions. Among these 66 SNPs, 43 (65\%) were novel and seven were located within regions corresponding to repetitive sequences predicted by the RepeatMasker program. Among the eight SNPs identified within coding elements, three were nonsynonymous and one, an Ala97Thr substitution (G289A) within the HEP27 gene, had not been reported before, while the remaining two (an Arg48His within $A D H 2$ gene and an Arg272Gln with $A D H 3$ gene) were identical to SNPs deposited in the dbSNP database (ID: rs1789884 and ID: rs1789912, respectively). With regard to the distribution of SNPs in each gene, we found 27 within the $27-\mathrm{kb}$ genomic region containing the $A D H 7$ gene but detected only three in the $20-\mathrm{kb}$ $A D H 5$ genomic region.

\section{Glutathione S-transferase family}

Glutathione S-transferases (EC2.5.1.18) are a unique group of multifunctional isozymes that play important roles in the conjugation and detoxification of various xenobiotics such as aflatoxins and polycyclic aromatic hydrocarbons. On the basis of similarities in amino acid sequences and crossreactivity of antibodies, mammalian cryptozoic glutathione S-transferases are divided into six classes: alpha, mu, kappa, theta, pi, and sigma. In addition, there is a class of microsomal glutathione S-transferases (for reviews, see Hayes and Pulford 1995; Strange et al. 2000). We analyzed a total of 12 glutathione S-transferase genes by direct sequencing (Fig. 1, g1-g12), and identified 127 SNPs: eight in coding elements, 22 in $5^{\prime}$ flanking regions, none in $5^{\prime}$ untranslated regions, 86 in introns, seven in $3^{\prime}$ untranslated regions, and four in $3^{\prime}$ flanking regions. Among these 127 SNPs, 104 (82\%) were considered to be novel because they did not overlap with SNPs in the dbSNP database, and 15 were located within regions corresponding to repetitive sequences predicted by the RepeatMasker program. Of the eight SNPs identified within coding regions, six were nonsynonymous and two, a Thr163Ala substitution (A487G) in the GSTA4 gene and a Val224Ile substitution (G670A) in the GSTM3 gene, were novel. Regarding distribution, SNPs were most frequent at the GSTM1 gene locus (21 SNPs were present in the $12-\mathrm{kb}$ region sequenced; i.e., one in every $480 \mathrm{bp}$ ), while only three SNPs were detected at the 36.5 -kb MGST2 locus (one in every $3000 \mathrm{bp})$. 


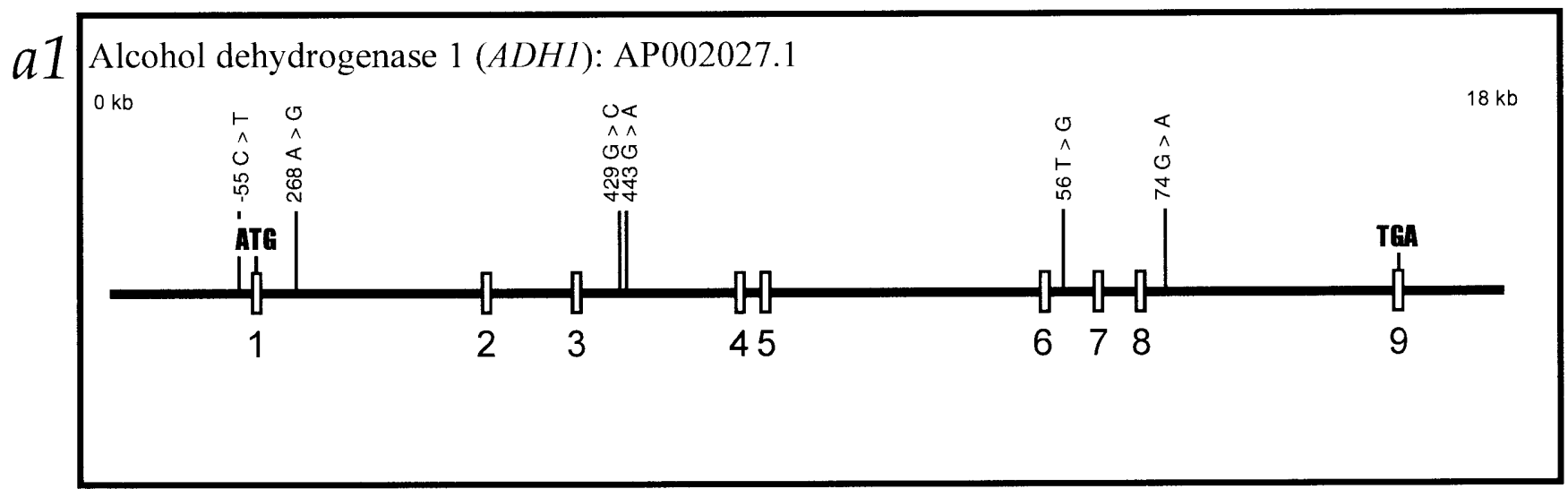

1 (5' flanking region -55)

atcatgtgtggaactggaat $\mathrm{C} / \mathrm{T}$ gggtgttattcaagcaaaa

2 (intron 1 268)

acatttgcggtaaagcgata A/G tttattccaagctaatcatg

3 (intron 3 429) dbSNP ID: rs1693437

4 (intron 3 443)

tgctgagttcacaaaatgga G/C gctacatggctat G/A

gctgaatgagcatgacctt

5 (intron 6 56)

tacaacttggaggatgcatt $\mathrm{T} / \mathrm{G}$ aggctgcagaatatatgttt

6 (intron 8 74)

gtctagcagaaaatgaaaag G/A tggaaggatgagaaaatta

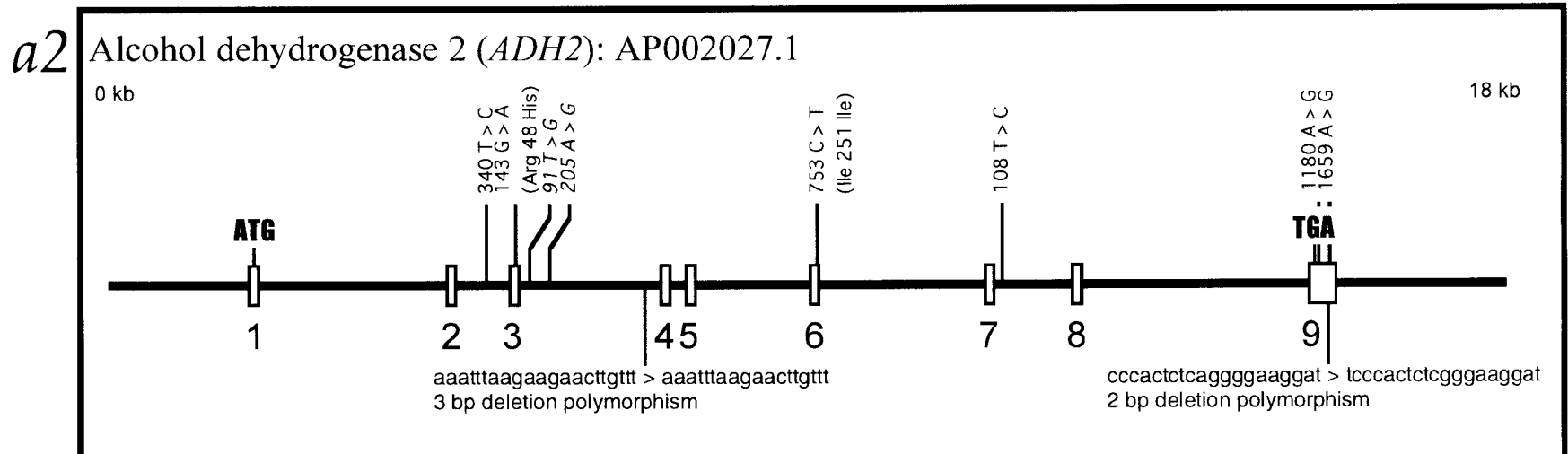

1 (intron 2340 )

ctattttttaaagcgtgcat $\mathrm{T} / \mathrm{C}$ cttacataagacttaaatat

2 (coding region 143) dbSNP ID: rs1789884

ggtggctgtaggaatctgtc G/A (Arg 48 His)

cacagatgaccacgtggtta

3 (intron 3 91)

4 (intron 3 205)

5 (coding region 753) dbSNP ID: rs1789882

aaggcaatgagagacgaaag $T / G$ gcttgcacaaggtcaccgcg

atgtattgtaccettcaacc $A / G$ ttatgtaccgagtatctact

caagactacaagaaacccat $\mathrm{C} / \mathrm{T}$ (Ile $251 \mathrm{Ile}$ )

caggaagtgctaaaggaaat

6 (intron 7 108)

acaattgacaaggcaagatt $\mathrm{T} / \mathrm{C}$ tgaaaacaaatcaaaataa

7 (3. untranslated region 1180) dbSNP ID: rs17033

ttcagcctcctctaccctac A/G agatctggagcaacagctag

8 (3' untranslated region 1659) dbSNP ID: rs1042026

tttacaagtagtgaaggtcc A/G agagttctaaatacaggaaa

Fig. 1. Genomic organizations and locations of single-nucleotide polymorphisms (SNPs) in 8 alcohol dehydrogenase genes (a1-a8), 12 glutathione S-transferase genes (g1-g12), and 18 NADH ubiquinone oxidoreductase genes (n1-n18). Exons are represented by open rectangles and introns by horizontal lines. SNPs are indicated above each gene, and microsatellite sequences, and insertion-deletion polymorphisms are listed below. For cDNA and coding sequences in genomic DNA, the adenine of the initiator Met codon is denoted nucleotide +1 . The first nucleotide of the $5^{\prime}$ untranslated region is numbered -1. Likewise, intronic DNA positive numbers start from the guanine of the donor-site invariant GT, and negative numbers start from the guanine of the acceptor-site invariant AG. Italic type indicates SNPs located within repetitive sequences predicted by the RepeatMasker program. The complete genomic structure of the NDUFA2 gene, determined for the first time in this study, has been submitted to the DNA Data bank of Japan under accession number AB054976.1 
a3 Alcohol dehydrogenase 3 (ADH3): AP002027.1

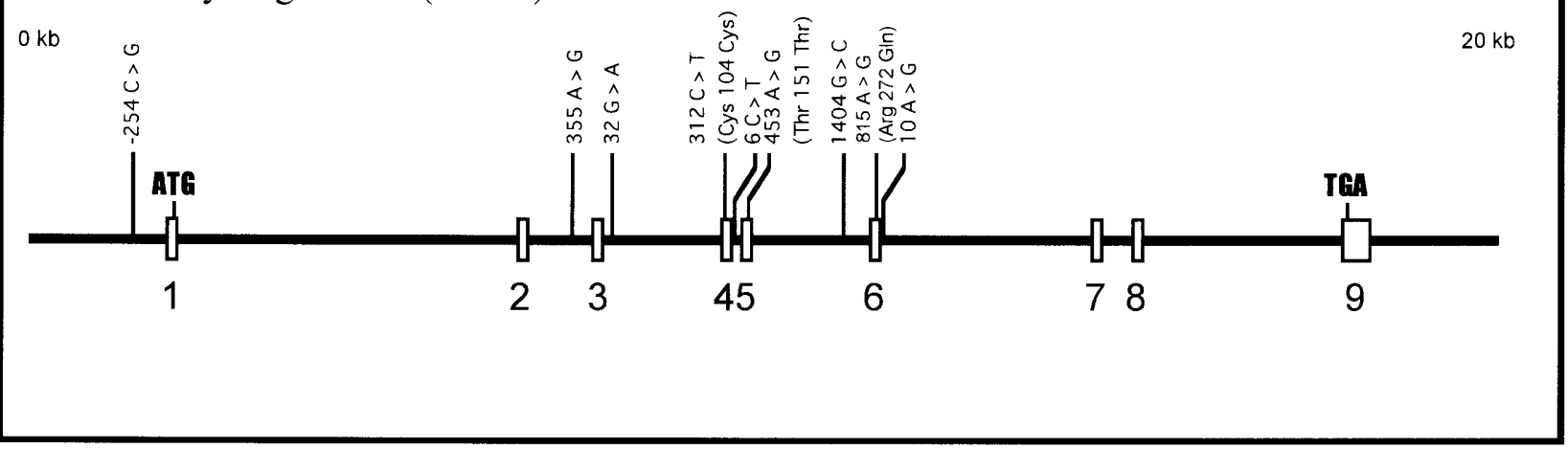

1 (5' flanking region -254)

2 (intron 2 355)

3 (intron 3 32)

tgagagaagagaagcaggaa $C / G$ ttgagagaggaggaagagag tatgcattcttctatattat $\mathbf{A} / \mathbf{G}$ caagacaaaatttaggat acactcagggaacatgcctt G/A gttcaccatcacaagattag

4 (coding region 312) dbSNP ID: rs1789915

tgtggaaatgcagaatttg $\mathrm{C} / \mathrm{T}$ (CYs 104 Cys) aaaac

5 (intron 46 )

6 (coding region 453 )

7 (intron 5 1404) dbSNP ID: rs1631460

8 (coding region 815 )

9 (intron 610 ) dbSNP ID: rs1789912

ctgcttgaaaaatgagtaag $\mathrm{C} / \mathrm{T}$ ttctgatgctttcttgcac

agcaccttctcccagtacac A/G (Thr 151 Thr) gtggtggatgagaatgcagt

gagatactgaagctggctat $\mathrm{G} / \mathrm{C}$ aagggatttcagcaattgag

ttcgtttgaagtcatcggtc A/G (Arg $272 \mathrm{Gln}$ ) gettgacaccatggtatgatcc A/G tgacatgccetgaaatt tct

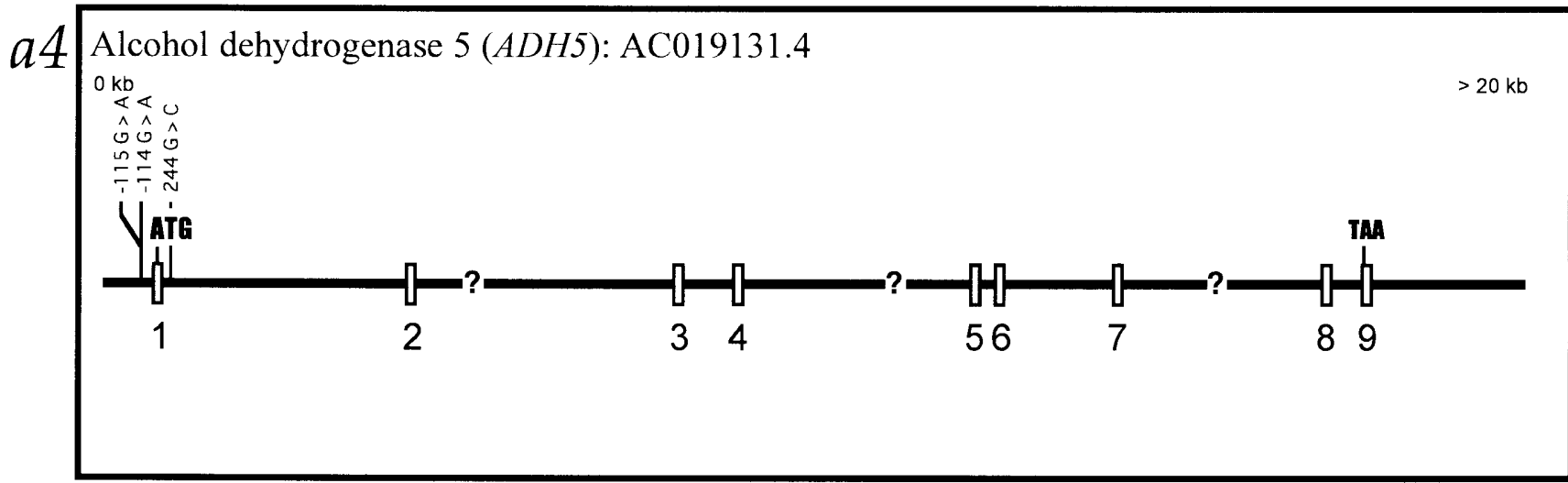

1 (5' flanking region -115)

2 (5' flanking region -114)

taactgctgtaaagttacac G/A G/A ggaagcctttcccgacaaa

3 (intron 1244 ) dbSNP ID:rs1154402 agtcggaacggggtgggggg G/C tgggagagccggagagtggg

Fig. 1. Continued 

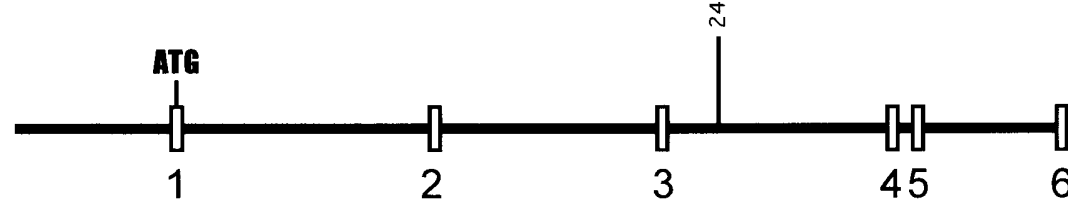

45
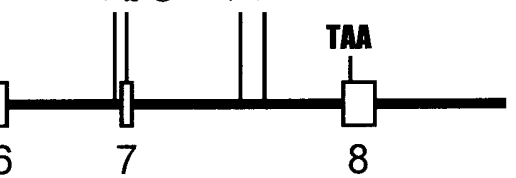

1 (intron 3 249)

2 (intron 6 1072)

3 (coding region 885)

4 (intron 7 1292)

5 (intron 7 1616) tgaaactggacttgaaagta $\mathrm{C} / \mathrm{A}$ aaatgagacaaaatttatg taaccctatactgtattgc A/G tcactttctaacaggcagct gtctgtgtggttgttggggt G/A (Val $295 \mathrm{Val}$ ) ttgcctgccagtgttcaact gttgagaaacactgcctagt $\mathrm{C} / \mathrm{A}$ ccgtctgtggtcctagaatt ctatcacagaataatccgca $\mathrm{T} / \mathrm{C}$ agaacactaagcagattacg

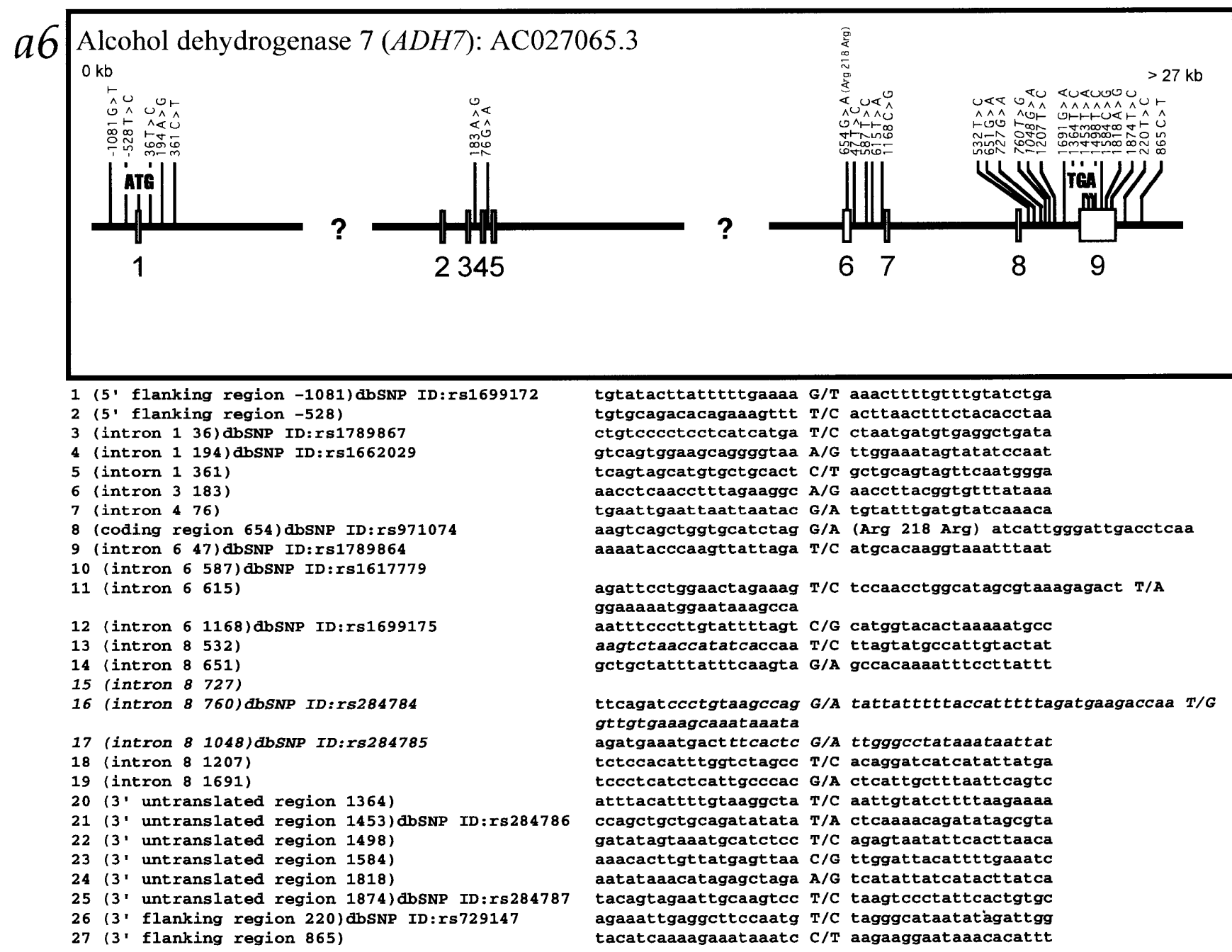

Fig. 1. Continued 
Hydroxyacyl-Coenzyme A dehydrogenase (HADH2): AL161779.20 $\mathrm{okb}$

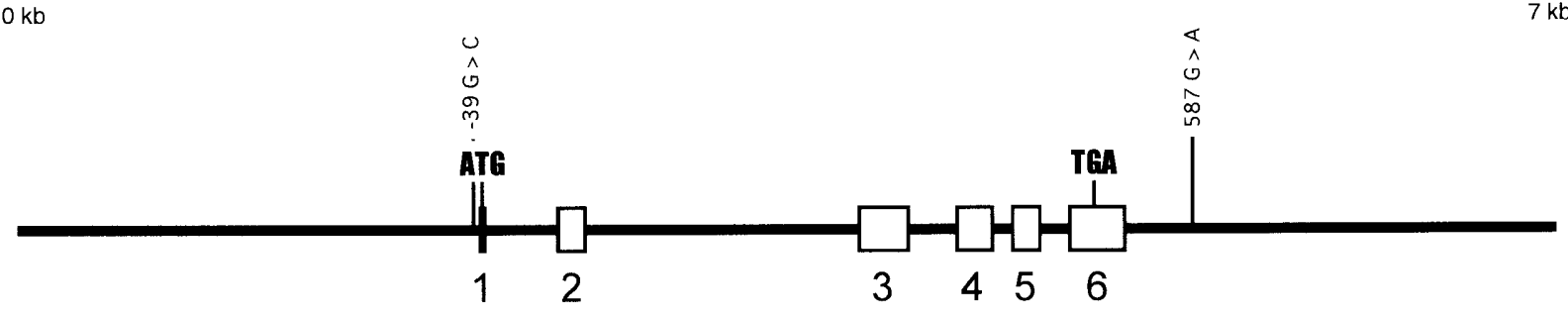

1 (5' flanking region -39) dbSNP ID: rs1264014

cgcatccgccecttccgccg G/C ttcgcctcggccaatcaacg

2 (3' flanking region 587) dbSNP ID:rs1264013 actgcctgggctgagctcat G/A gtctggcttttccattcagt

Short-chain alcohol dehydrogenase family gene (HEP27): AL135999.3

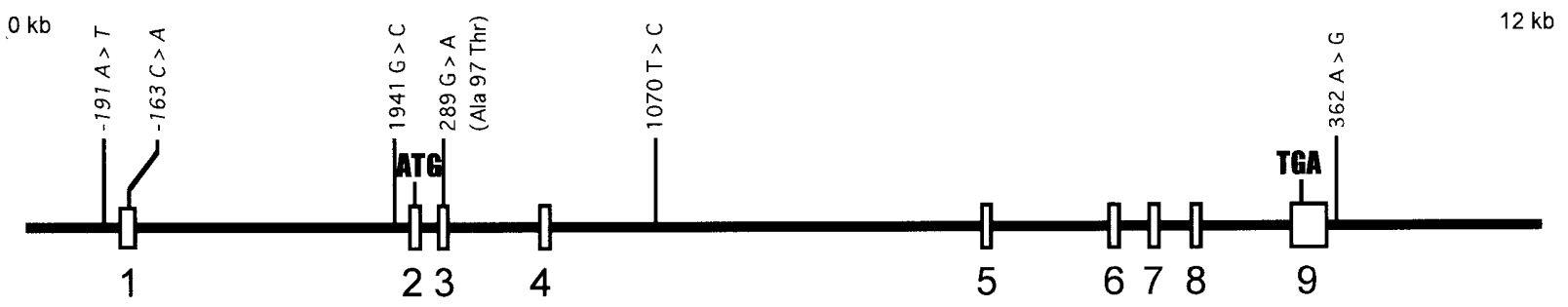

(5' flanking region -191)

tcagcactctgtgtctagct $A / T$ aaggtttgtaaatgcaccaa

2 (5' untranslated region -163)

gaacccatcaattccgtaca $\mathrm{C} / \mathrm{A}$ attttggtgactttgaagag

3 (intron 1 1941)

aatttaccctaaccagcct $\mathrm{G} / \mathrm{C}$ actctctgccactttctgtt

4 (coding region 289)

5 (intron 4 1070)

6 (3' flanking region 362) ttgtgtgccacgtggggaag G/A (Ala $97 \mathrm{Thr}$ ) ctgaggaccgggagcagctg tgtctcagttcacaggatca $T / C$ gactcttttctcgaaactg ggctttgtgtgtgctccatt A/G tctgaactgggcetgctggg

Fig. 1. Continued 


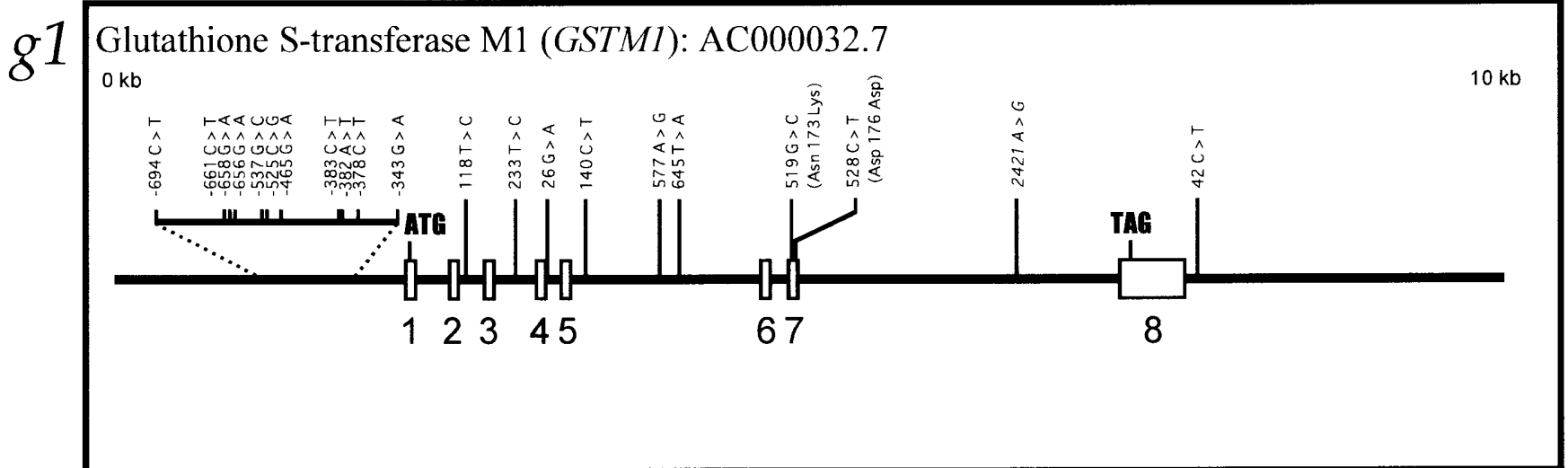

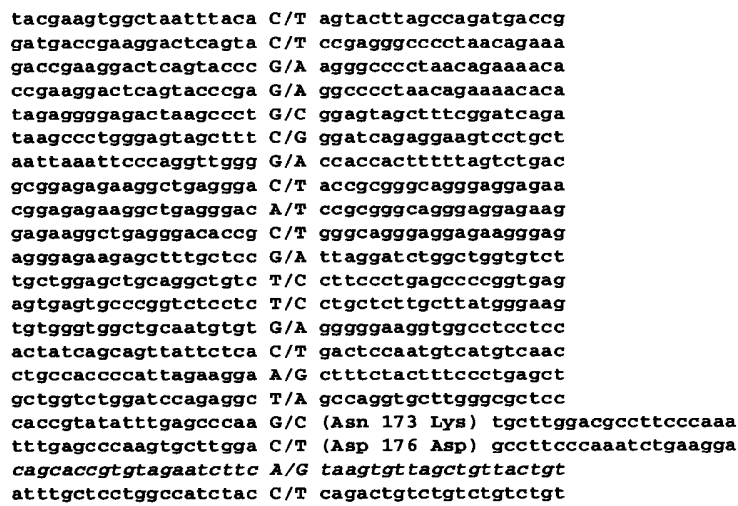

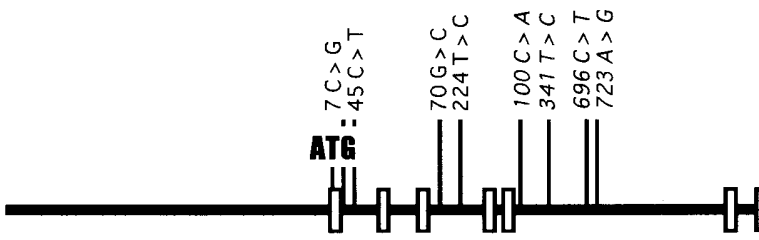

$12345 \quad 67$
1 (intron 17 )

2 (intron 145 )

3 (intron 3 70)

4 (intron 3 224)

5 (intron 5 100)

6 (intron 5 341)

7 (intron 5 696)

8 (intron 5723 )

9 (intron 7 2836) abSNP ID: rs1149179

10 ( 3 , untranslated region 1006)

11 (3) flanking region 139) ggaacatccgcggggtgagc C/G agggtccgctgggcggtggg gggacgggggtgcgtggggg $C / T$ ggggaagtgtggagcagctg gactgcatctcctctcccca G/C cttagaggtgttaagatcag agcaggccctggtctcctct $\mathbf{T / C}$ tgccettgcatatgggaagg ttgattccttctggtgagtt C/A ttggtcttgctgactctaag tcctcttggtgggttcatgg $T / C$ ctggctggcttcaggagtga acctttagctagacacagag $C / T$ gctgatttgtgcatttacaa ttgtgcatttacaatccttt $\mathrm{A} / \mathrm{G}$ gctaggcagaaagttctcc aacttctgtttcccacatga $C / G$ aatggtgataatagattca ctcagccccgagctgtcccc G/A tgttgcatgaaggagcagca ttctgctgggcatagtaagg $C / T$ gcttgagaattcttgctccc

Fig. 1. Continued 
$g 3$ Glutathione S-transferase M3 (GSTM3): AF043105.1

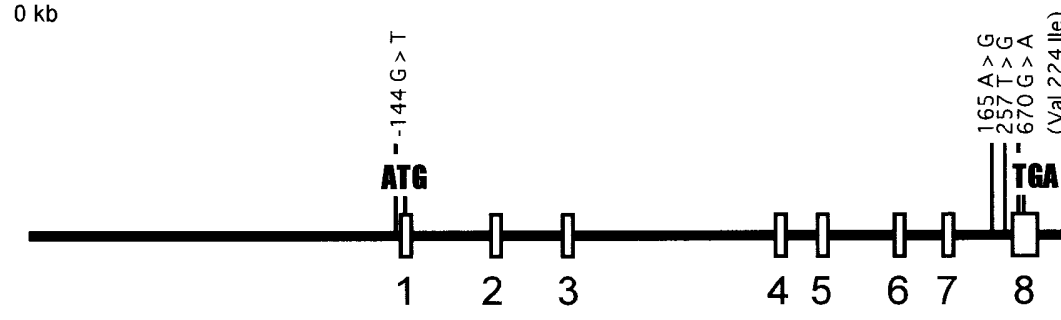

1 (5' flanking region -144)

2 (intron 7 165)

3 (intron 7 257)

4 (coding region 670) ccaacgccggcattagtcgc G/T cctgcgcacggccetgtgga agcctaacttctataccttg $\mathrm{A} / \mathrm{G}$ aggcactgtctacaaaaaa ctgttggactgggtggggtc $\mathrm{T} / \mathrm{G}$ ttataagattggtgtattt cccagtggggcaacaagcet G/A (Val 224 Ile) tatgctgagcaggaggcaga

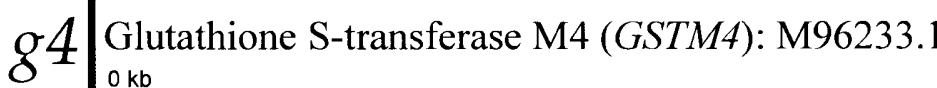

$u$
$\hat{\imath}$
$\hat{\circ}$

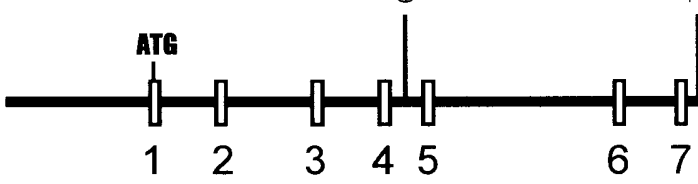

$\begin{array}{lll}0 & \varangle \\ \wedge & \hat{N}\end{array}$

$\hat{\wedge}$

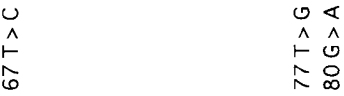

$\begin{array}{lllll}1 & 2 & 3 & 4 & 5\end{array}$

67

8

1 (intron 4 67)

2 (intron 7 77)

3 (intron 780 ) ttggctggattggggtgcta T/C gctcagagtgagtctgtgtt

gatgctttcccagtcctgga T/G ct G/A cataagaataacttgcatt

Fig. 1. Continued 


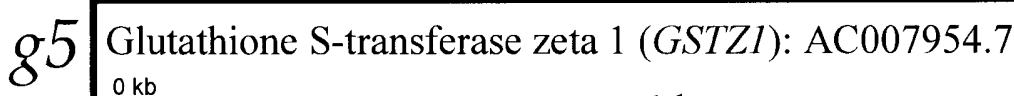

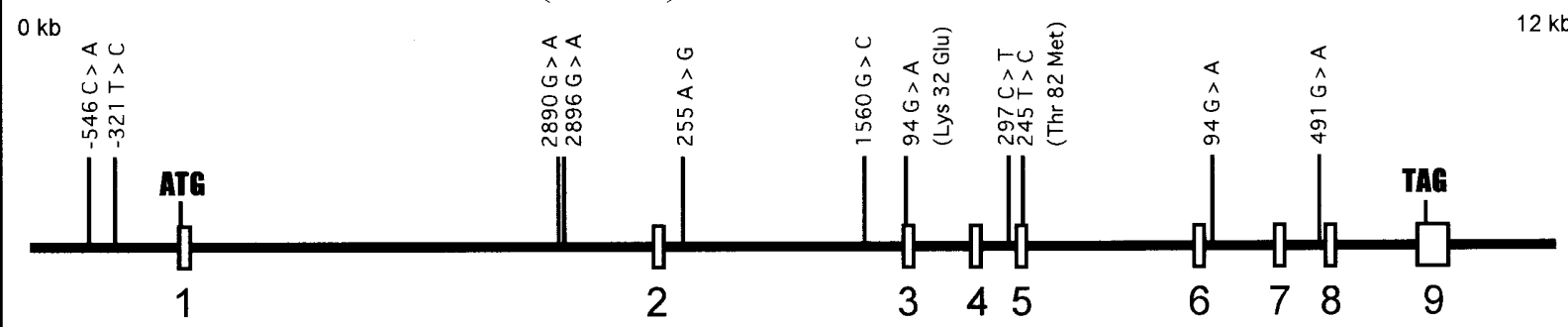

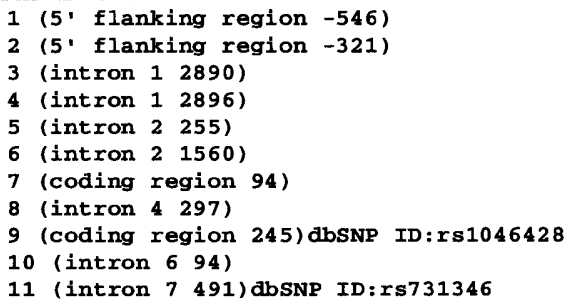

agcagggcccaccagccgac C/A gcctcgaagcgcegtgagcc tgtctgaccagccgccccgc $T / C$ aaggagtcacaagagggcag aaatactgcatcaaaacca G/A gccacgctctgttgggggga ctgcatcaaaccaggccac G/A ctctgttggggggacaccaa tctcccaacactgctctcca $A / G$ agcccttggcaaccatgtt caccactgtttaaggccetg G/C gggggcagagttaaacacaa

ccttgaaaggcatcgactac G/A (LYs $32 \mathrm{Glu}$ ) agacggtgcccatcaatctc agaaggaggagtttgctggc $\mathrm{C} / \mathrm{T}$ ctgtcccctctggtccaggg cattgagtatctagaggaga T/C (Thr 82 Met) gcgtcccactccgcgacttc tatctgaaccagcctcccag G/A ctgctttgggcctgacagtt ccctgatgggaacccaccgg G/A acctcttcaggtgaccatg

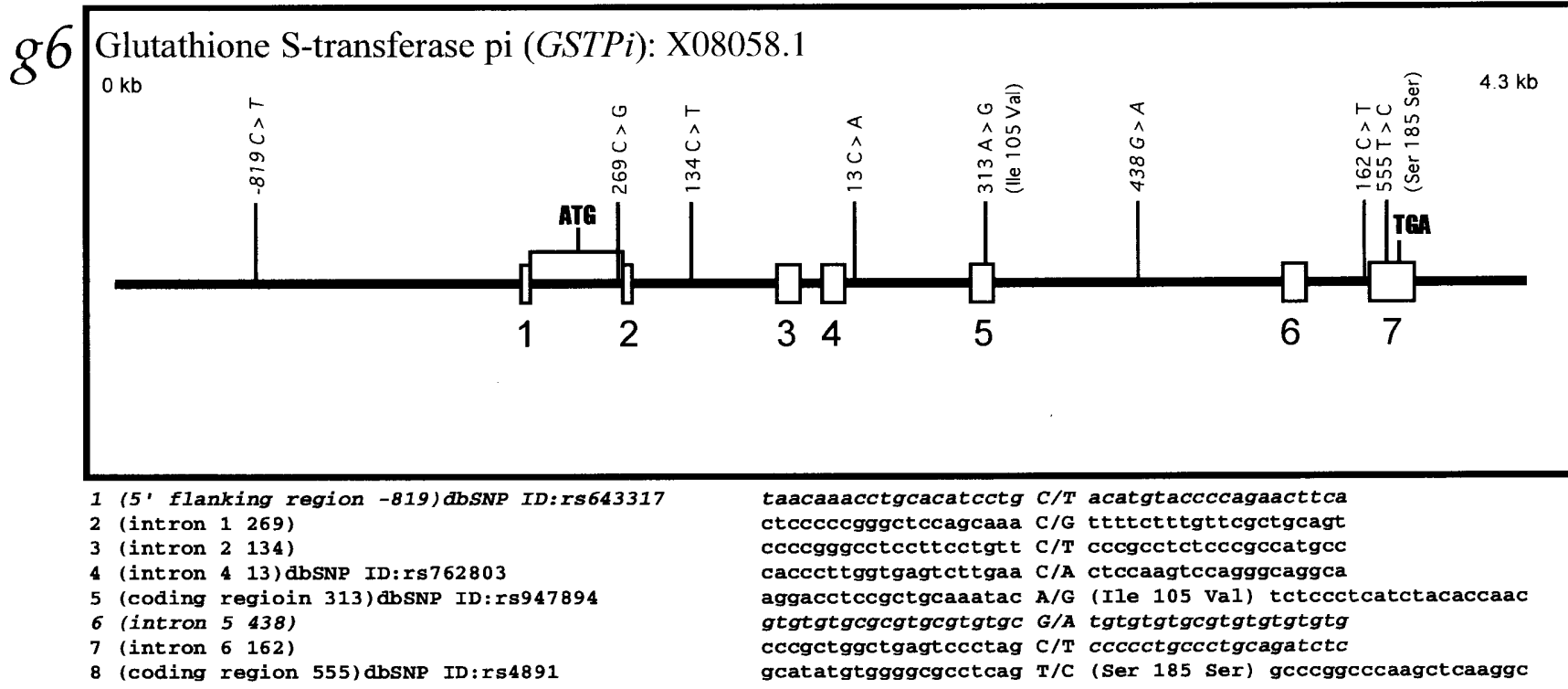

Fig. 1. Continued 


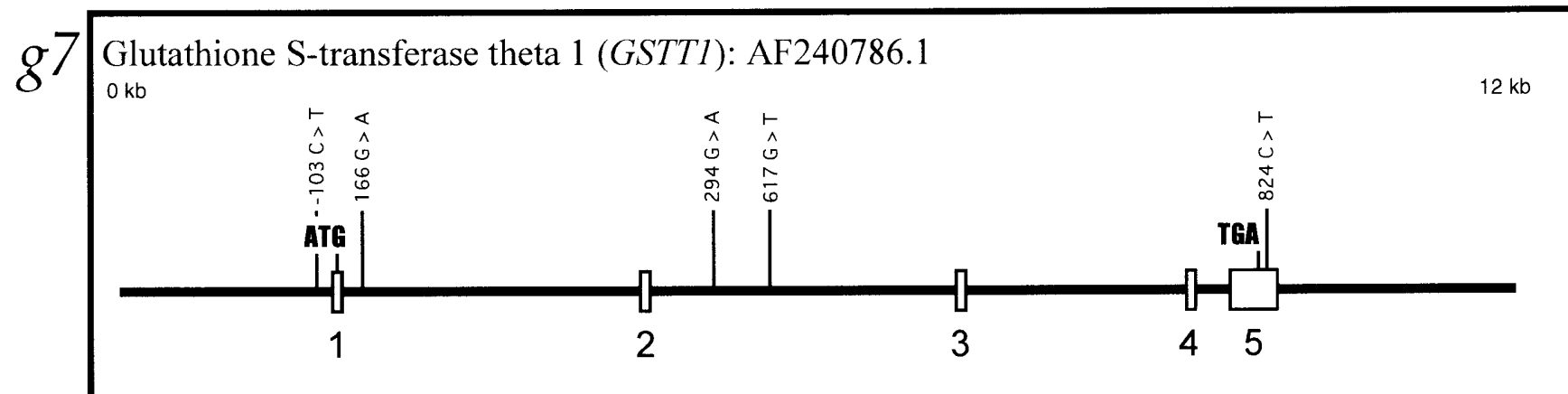

1 (5' flanking region -103 )

2 (intron 1 166) dbSNP ID: rs140313

taaagagtgtcccaggcgtc C/T gtgccgcccaatggggcaca

3 (intron 2 294) dbSNP ID: $r S 140310$

agcagggagatccaagagtc G/A gggctccccaaactctgct

4 (intron 2 617) dbSNP ID:rs140309

cgatggcagctttgcccacc G/A tggggcaggcctctggccaa

5 ( 3 ' untranslated region 824) dbSNP ID:r\$4630

ctgacattctgccagggcc $G / T$ tcttcctcctggtgcccca agaaagcaggaatggcttgc $\mathrm{C} / \mathrm{T}$ taagacttgcccaagtccia

g8 Microsomal glutathione S-transferase 1-like 1 (MGST1L1): AC007936.2

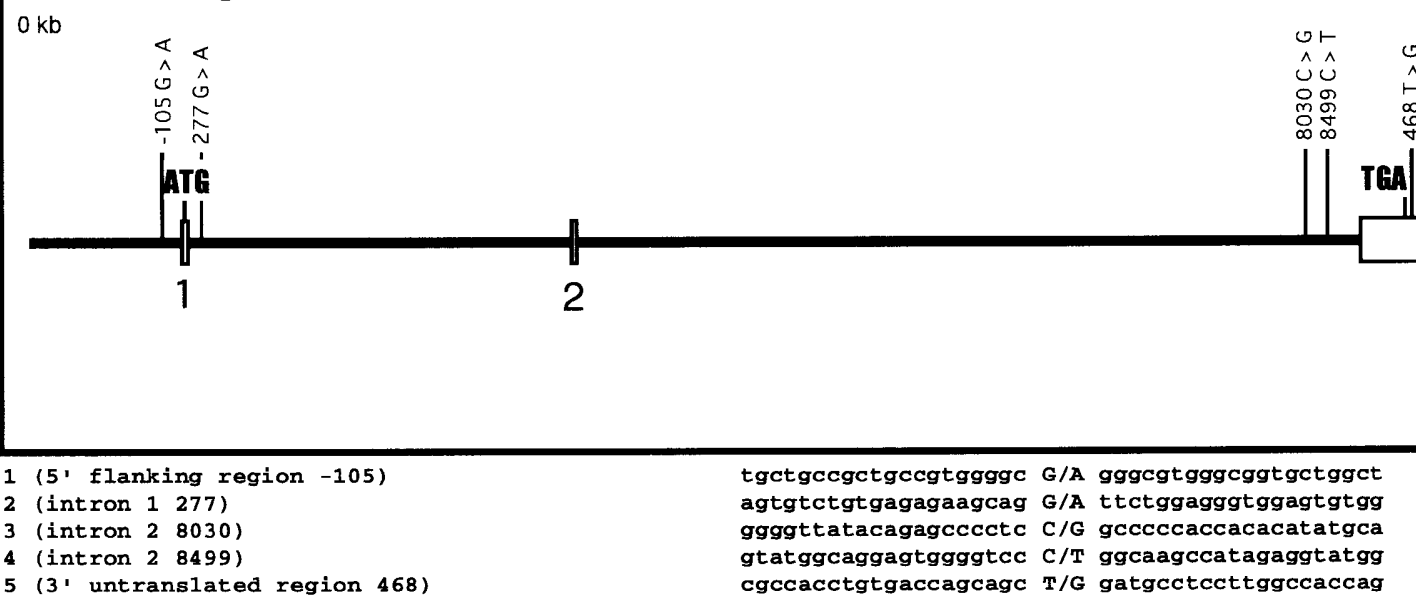

Fig. 1. Continued 


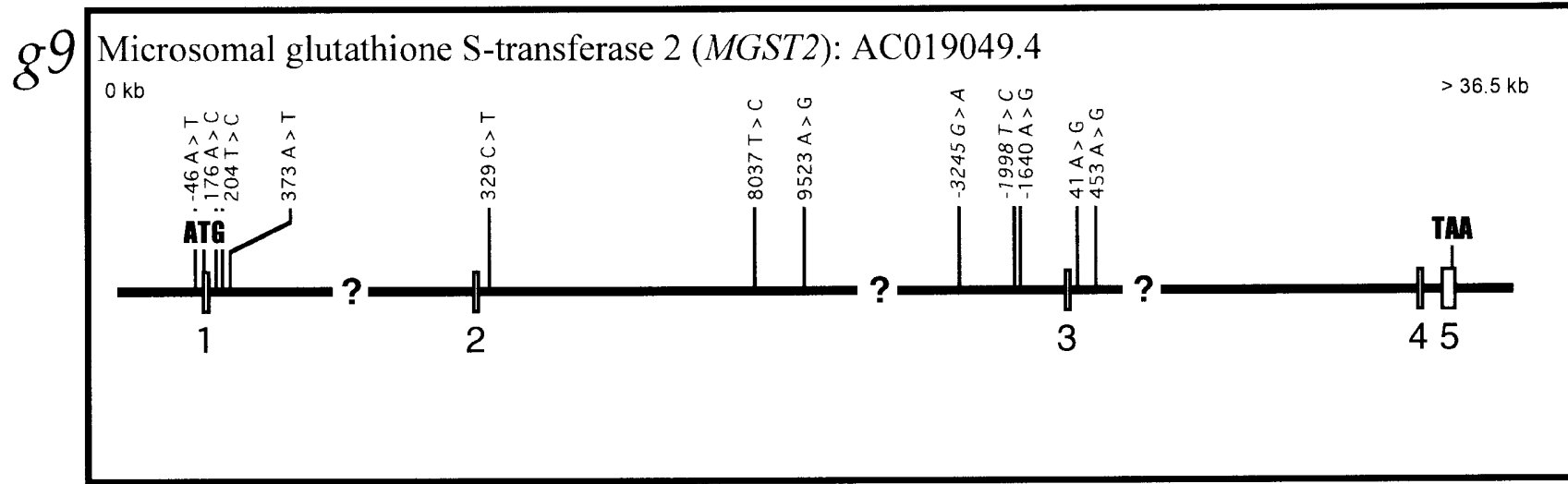

1 (5' flanking region -46 )

2 (intron 176)

ggtcagcattcaaagtcaag $\mathbf{A} / \mathbf{T}$ agcgccattatcttccegt

(intron 1204 )

4 (intron 1373 )

5 (intron 2329 ) dbSNP ID: rs795589

6 (intron 2 8037) dbSNP ID: rs1453135

7 (intron 2 9523) dbSNP ID: rs1377382

8 (intron $2-3245$ )

9 (intron 2 -1998)

10 (intron $2-1640$ )

11 (intron 341 )

ggtcacccatgccgcctgct $\mathrm{A} / \mathrm{C}$ ccctccttcccaggggcaag

tcccaggggcaagcagagac $\mathrm{T} / \mathrm{C}$ gagaacattccagagattag

ttacaagtgttccaaaggaa $\mathbf{A} / \mathbf{T}$ cgtgcctgcttctaacctg

agaccacaatagtctgaagt $C / T$ ggattagttatgctacaatt

aagctacactcttctgct $\mathrm{T} / \mathrm{C}$ tgtaatgatgtaaattat

ctgaaagaccattattaagc $\mathbf{A} / \mathbf{G}$ tcttcatcttctggattt

cctcgtgatttgcccacctc G/A gcctcccaaagtgctgggat aggccgaggtgggcggatca $T / C$ gaggtcaggagatcgagace tgtttattccttgcatagc $\mathbf{A} / \mathbf{G}$ taatataagtatgaattt actgtgttctaatgatgact $\mathrm{A} / \mathrm{G}$ tgatgcttaaacgattaagg

12 (intron 3 453)

atcagagtgctatgttgcag $\mathrm{A} / \mathrm{G}$ tatatgaactttggettcat

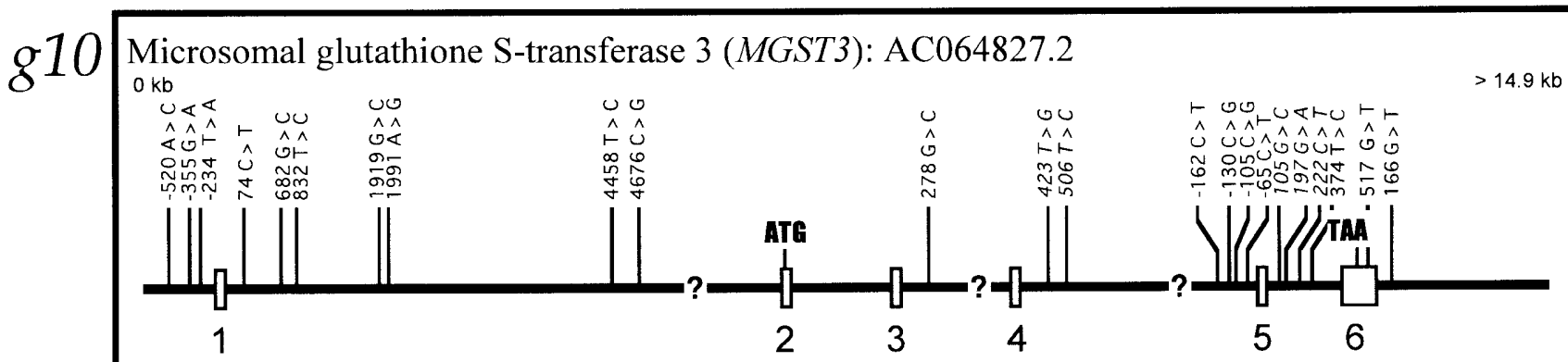

1 (5' flanking region -520 )

2 (5, flanking region -355 )

3 (5' flanking region -234 )

4 (intron 174 )

5 (intron 1682 )

6 (intron 1832 )

7 (intron 1 1919)

8 (intron 1 1991)

9 (intron 1 4458)

10 (intron 14676 )

11 (intron 3 278)

12 (intron 4423 )

13 (intron 4 506)

14 (intron $4-162$ )

15 (intron $4-130$ )

15 (intron $4-130$ )

16 (intron $4-105$ )

17 (intron $4-65$ )

18 (intron 5 105)

19 (intron 5 197)

20 (intron 5 222)

21 (intron 5 374)

22 ( 3 ' untranslated region 517)

23 ( 3 , flanking region 166) acaaaaggccetaacagcg $\mathbf{A} / \mathbf{C}$ taatccattcacttcggga cgcctaaaccgctacggtg $\mathrm{G} / \mathrm{A}$ ctctgctggggacaaat tat ctgggggagtagatatatgt $T / A$ tttgagaatgagaggagtaa agcctttgcgcaggcactcc $C / T$ atattcagcctatgcgagc agaaat geccettctttat $G / C$ tggggtggcagcacggagce gaaatgccctecttat G/C tggggtggeagcacggagce gagttracagctacataa T/C agcgtcogoggcaagtaat aataaattcctgagttect G/C tcactegetettacagtacc tgtaattaggcaacaggaaa $\mathrm{A} / \mathrm{G}$ tegtactatcttcaaatgc tcttccatcctcctaacata $T / C$ agttagcttccactctcca tgaatatgcaatgcaattgt C/G gggggatagttactttea cagcatgacccatctaaacc $\mathrm{G} / \mathrm{C}$ atgttgactctcccaggcct cttgcctttttgttgtgggg $T / G$ gtggggtggtcacagagaag gtgcagagaagaaacaaag $T / C$ ggggaaggtggaaaggggat tcacagatattt tattt $\mathrm{CCC} C / \mathrm{T}$ gactgaaactaact taatt acttaat cctacctaatttg $\mathrm{C} / \mathrm{G}$ gtggggagtagttggccaa gottaattctacctaatetg C/G grgggagtagttggccaa gagete te te atcccagcactetgggaggc $\mathrm{G} / \mathrm{C}$ aaggcaggcagattgcttga aaaaatacaaaattagCC $G / A$ gatgtggtggtgcacactg tggtggtgcacacctgtagt $C / T$ ccagctact tgggaggctga tcttatgctactatattttt $T / C$ ttcttgggaatt tgagaaa atgacttaccttatttcca $\mathrm{G} / \mathrm{T}$ ttacattetttetaaata agtctgattgtggtgatgta $\mathrm{G} / \mathrm{T}$ gtatagtcatgccacagtga

Fig. 1. Continued 
$g 11$ Glutathione S-transferase A1 (GSTA1): AC021133.4

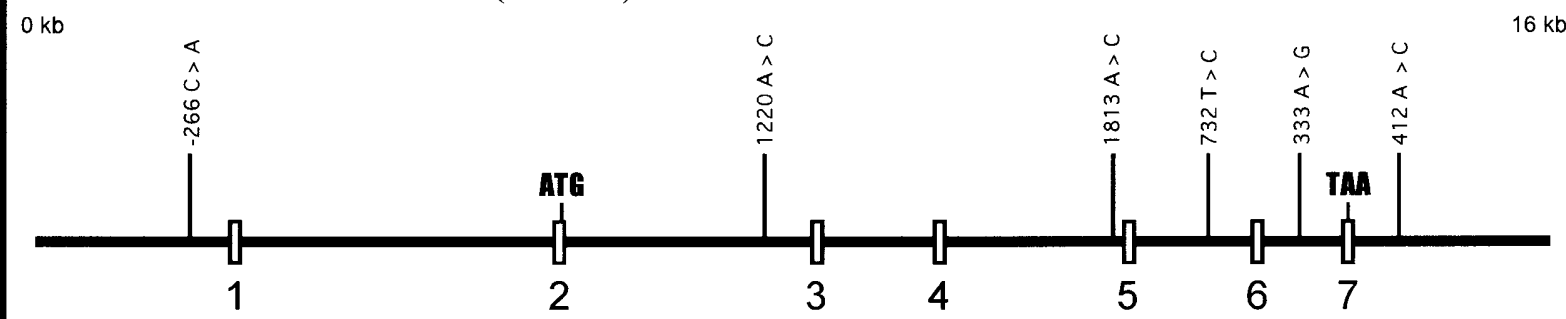

1 (5' flanking region -266 )

ttgcaaaaagagcaaaatct $\mathrm{C} / \mathrm{A}$ ggtgaaatgtattgtgtaaa

2 (intron 2 1220)

3 (intron 4 1813)

4 (intron 5 732)

gagacacaggctttcctaag A/C tatgacaacaccataactag

aaaggcacccactggaggtg $\mathrm{A} / \mathrm{C}$ attatttgccatcacctga

gaagagtgttgtcatgaagg $\mathrm{T} / \mathrm{C}$ ggagtcactgcccaagggag

5 (intron 6 333)

ttatcccatatgtgcccaca A/G tgagccggtctgagcagagc

6 (3' flanking region 412 )

ctttcttatgcatttgcaaa $\mathrm{A} / \mathrm{C}$ caatgattctgtctgctgtg

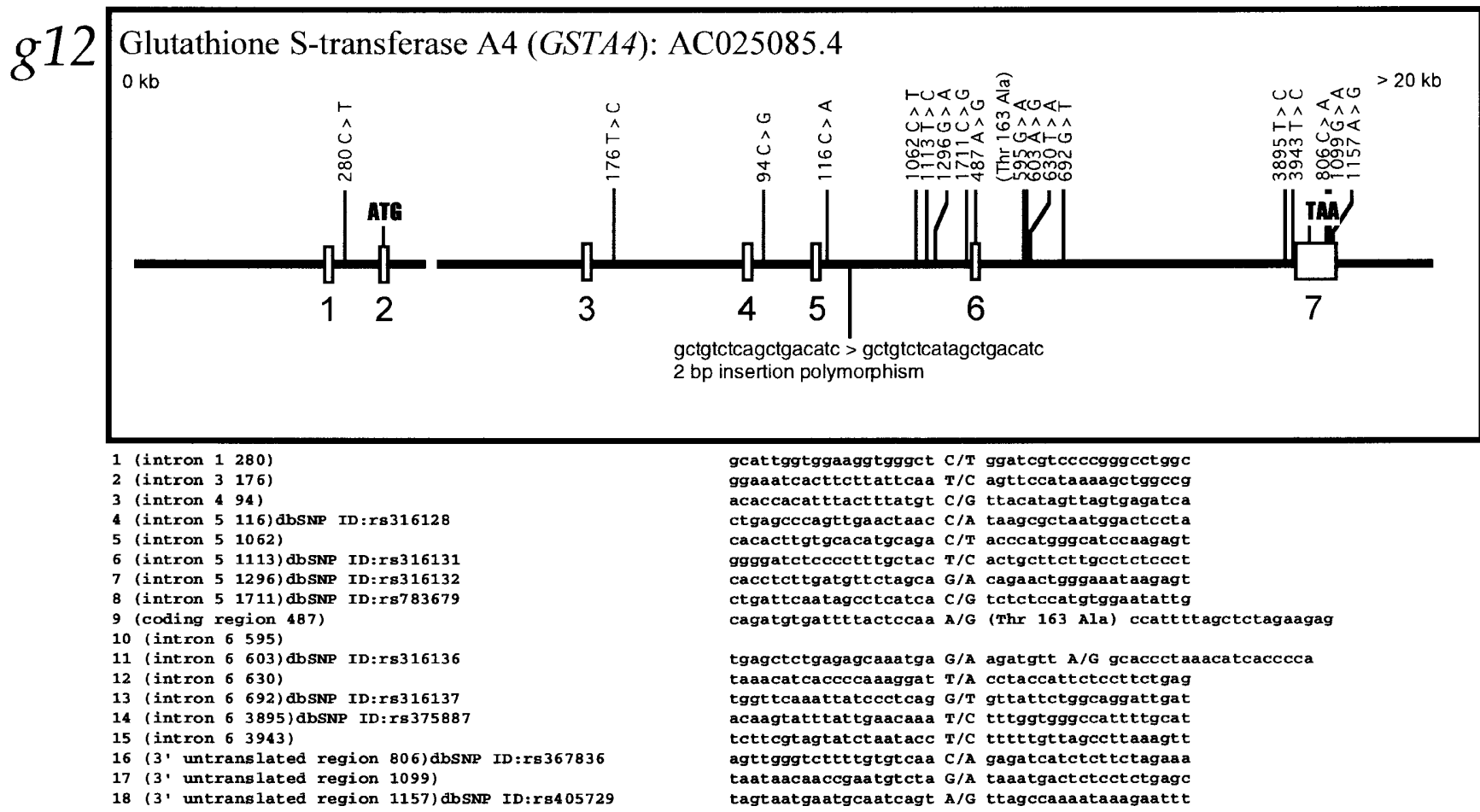

Fig. 1. Continued 
$n 1$ NADH ubiquinone oxidoreductase 1 alpha subcomplex 1 (NDUFAl): AC002477.1

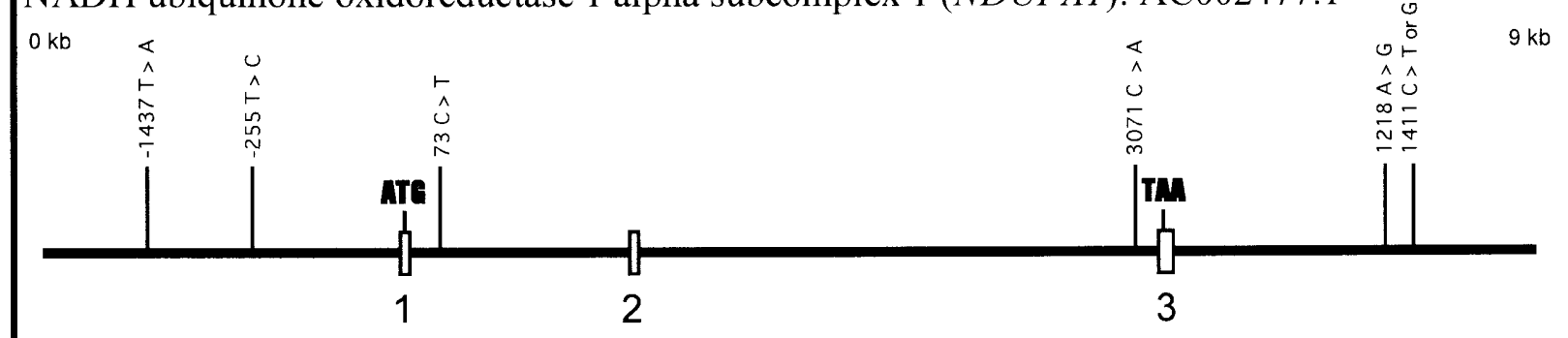

1 (5' flanking region -1437)

agggctaaaaatcctgatta $\mathbf{T} / \mathbf{A}$ acctaccttgaagctttaa

2 (5' flanking region -255) dbSNP ID: rs 1800823

3 (intron 1 73) dbSNP ID: rs708463

gctctgccattttagagtcc $T / C$ gagctccgaaacagccgtgg

4 (intron 2 3071)

gtggtgggcagggagaccgt $\mathrm{C} / \mathrm{T}$ agcctgcgaaccctctctcc

5 (3' flanking region 1218)

aataaaagtacatggcatat $\mathrm{C} / \mathrm{A}$ tttgatgggaacagacttgt

aactccatgtgtataagca $\mathbf{A} / \mathbf{G}$ caccacagatgacacttcca

tri-allelic

6,7 (3' flanking region 1411) ggattgtgccatcccttgat C/T/G ggcaatgacctttactttt

$n 2$ NADH ubiquinone oxidoreductase 1 alpha subcomplex 2 (NDUFA2): AB054976.1

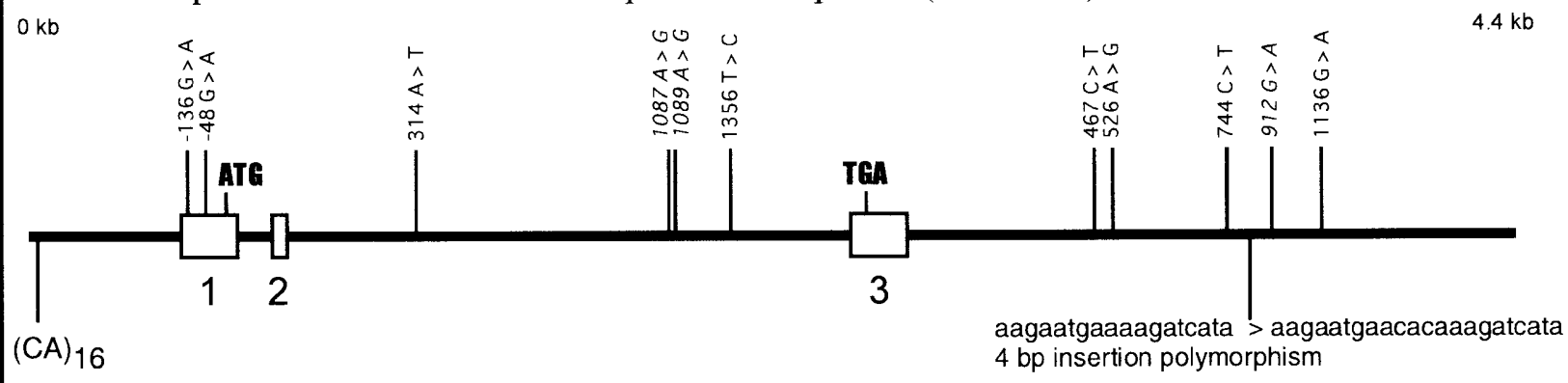

1 (5' untranslated region -136) dbSNP ID: rs778594

cctcgcagcggtcctacaat $\mathbf{G / A}$ ctttatatcattggccaag

2 (5' untranslated region -48) dbSNP ID:rs778593

tccgcggttggtcagaccgg G/A gcacttggcctgaagacctg

3 (intron 2314 ) dbSNP ID:rs778592 tccaaataacgtgtagccag A/T gtcagtccaggtagtgtgtg

4 (intron 2 1087)

5 (intron 2 1089)

aacatacaaaaattagccgg $A / G$ t $A / G$ tggtggcgggcacctgtaat

6 (intron 2 1356)

ttccctgaaacaacccattg T/C ggccatccagaatcagccaa

7 (3' flanking region 467)

cacagcctcatgggtcagcc $\mathrm{C} / \mathrm{T}$ actccagagggtgcattccc

8 (3' flanking region 526) dbSNP ID:rs702399

9 (3' flanking region 744)

tctgacatggaacctatata A/G gttagccaggccagagaaa

10 (3' flanking region 912) dbsNP ID:rs778590

catgccaagctcttctacat $G / A$ catgttctcatactcctaca

11 (3' flanking region 1136) dbSNP ID: rs753279

actgctgtcccatacaatcc G/A actaccagatcttggattca

Fig. 1. Continued 


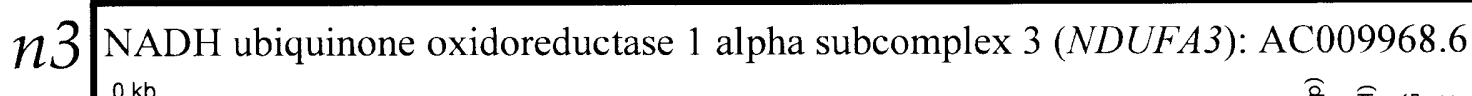

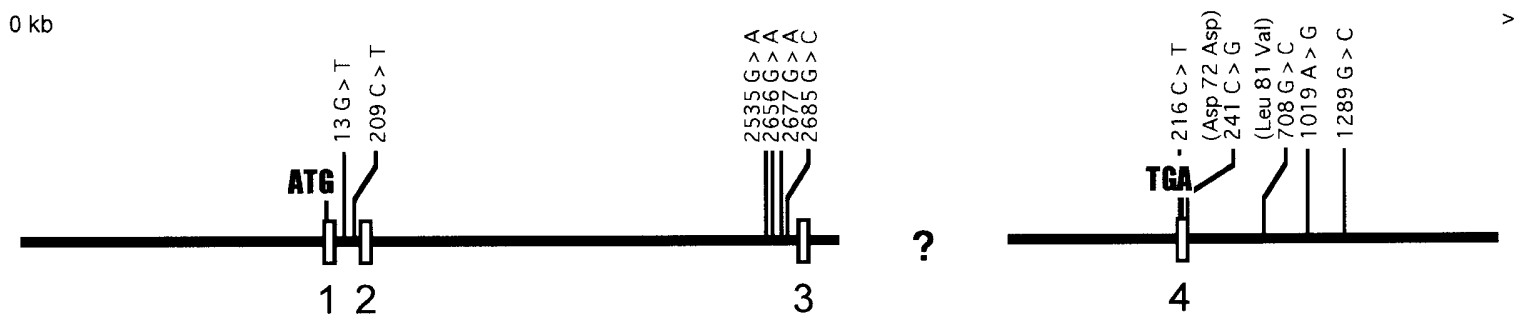

1 (intron 1 13) dbSNP ID:rs254260

2 (intron 1 209) dbSNP ID:rs254259

3 (intron 2 2535) dbSNP ID:rs254257

4 (intron 2 2656)

5 (intron 2 2677) dbSNP ID:rs254256

6 (intron 2 2685) dbSNP ID:rs254255

7 (coding region 216) dbSNP ID:rs1061333

8 (coding region 241 )

9 (3' flanking region 708 ) dbSNP ID: rs254264

10 (3' flanking region 1019)

11 (3' flanking region 1289) dbSNP ID: rs1193923 ggctgcgagtaagtgcaggt G/T ccggtggcgcacggggctcg ctacttgcaggggtgacgct $\mathrm{C} / \mathrm{T}$ cttgccacccttcaggagt atctgtgagtgttaggctcc $\mathbf{G / A}$ acccctacctccacttaacc tccetgctgccetcccetgc G/A cactttatcttccetttgcc

cacttatcticcittgcc G/A aggctca G/C cttctcttcc cetctctea

gtgcccagccaccccagga C/T (Asp 72 Asp) cctcagggce ccagcetgga

agggccccagcctggagtgg C/G (Leu 81 Val) tgaagaaact gtgagcacct

cccacactgggcettccett $\mathrm{G} / \mathrm{C}$ cactccgtcttctctgatcc tccttacctgcactggcace $\mathrm{A} / \mathrm{G}$ gctctggagcccagtccet $n 4 \longdiv { \substack { \text { NADH ubiquinone oxidoreductase } 1 \text { alpha subcomplex } 5 \text { (NDUFA5): AC073323.5 } \\ 0 \mathrm { kb } } }$

getgccetcatcctggcagg G/C aggagggggaggtaggtgat

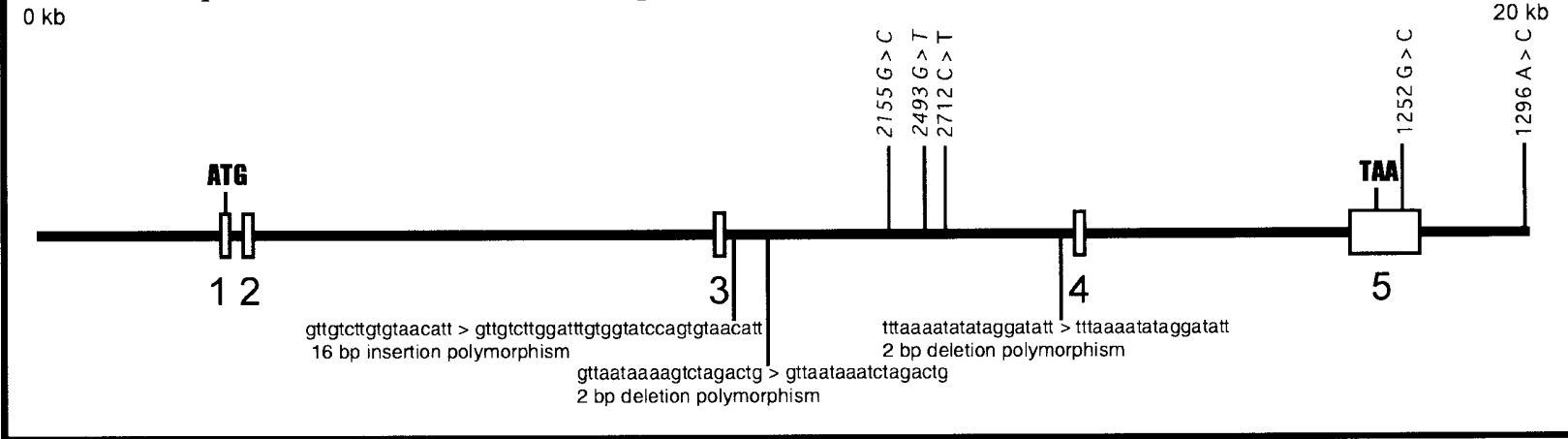

1 (intron 3 2155)

2 (intron 3 2493)

3 (intron 3 2712)

4 (3' untranslated region 1252) dbSNP ID:rs6737

5 (3' flanking region 1296) agactctagcatggtacctg G/C aacataaggttccttagaaa ggcatattgctagtttctc $G / T$ gtctcaattcatcatctat acaaattttgaactgttcac C/T taacacaggctttttctgaa gaaattatatctggcatac G/C gataggcttatattcaaaac aggtatctaaaggtattgc $\mathbf{A} / \mathrm{C}$ atttggtcattggttctttc

Fig. 1. Continued 
$n 5$ NADH ubiquinone oxidoreductase 1 alpha subcomplex 6 (NDUFA6): AL021878.1

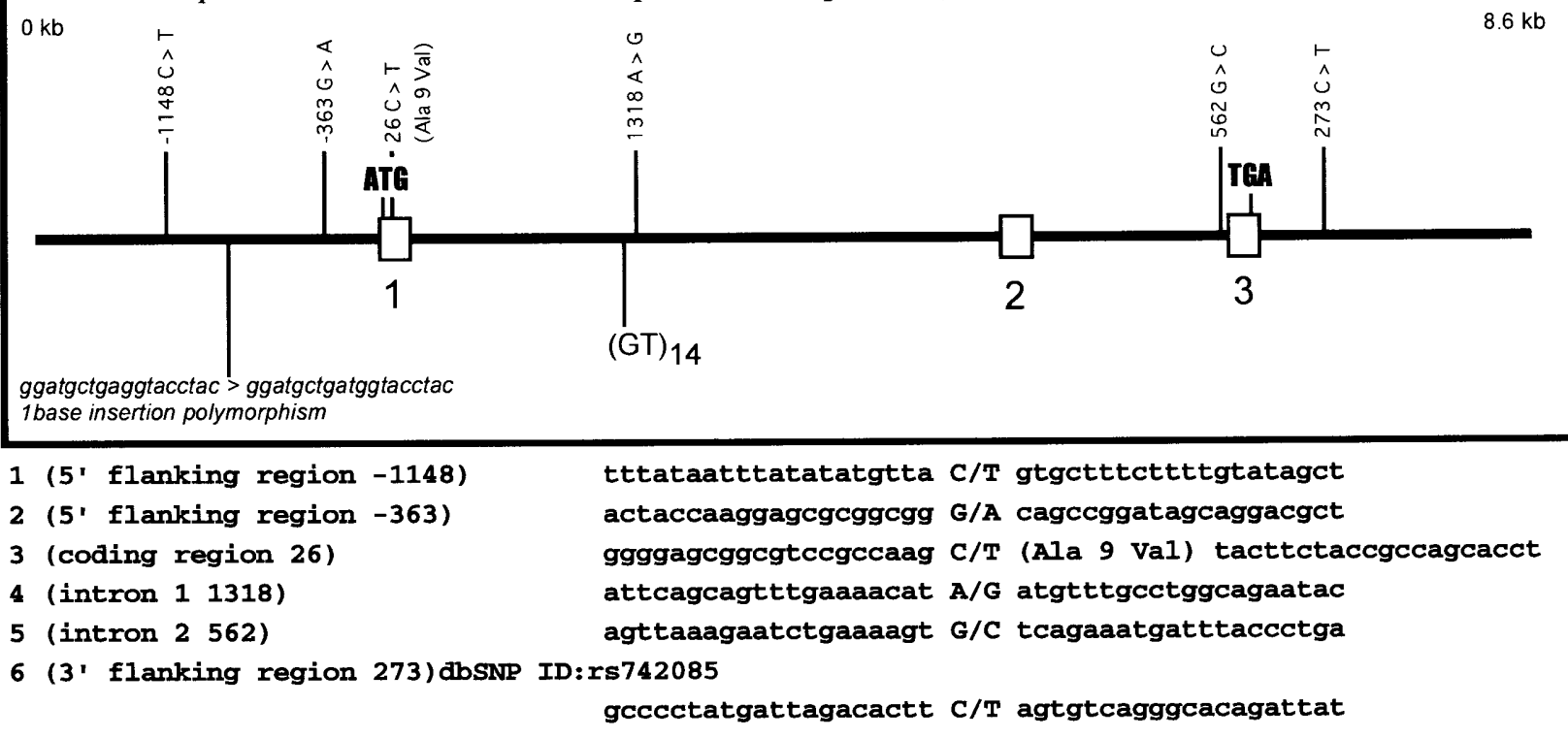

$n 6$ NADH ubiquinone oxidoreductase 1 alpha subcomplex 7 (NDUFA7): AC010323.6

1 (5' flanking region -940) dbSNP ID:rs1057232

ggacaaaaaaaggagcaga $\mathrm{C} / \mathrm{T}$ tgtgacagaccattcccatc

2 (5' flanking region -731) accaaccaaaggtctatcaa A/G ggggtgtcctctttgcaccc

3 (5' flanking region -434) aaagggaaccatcagaaccc C/T gtgatgaaatgagaatcggc

4 (5' flanking region -395) gctcccggattccggctggc A/G ggggttagggcagggtagag

5 (5' flanking region -100) agaggagtcacgtgcttcgg G/A gagagcctttataggacgtt

6 (intron 1 92) tcacctccctcctaagccgg G/A acccttcgctctcccogat

7 (intron 1 133)

8 (intron 1 136) ctccetgggaacccccagct $\mathrm{A} / \mathrm{C}$ gt $\mathrm{C} / \mathrm{G}$ acccettcagcccgggaccc

9 (intron 2 89) tcctttagaccctgaaacg G/C agggctgacatcctgccacc

10 (coding region 196) gccgccgggaatctgtgccc C/G (Pro 66 Ala) cttccatcatcatgtcgtcg

11 (intron 3 4203) gcctccaccctggggcgcc $T / G$ cctccatcacccacctcc

12 (intron 3 4604) gggccttgtgtacgctggag A/G ccaaaagtgggaagggagga

13 (coding region 300) dbSNP ID: rs1045629

gcggtgactccagctcctcc C/T (Pro 100 Pro) ataaagaggtgggagctgtc

14 (3' untranslated region 354) dbSNP ID: rs561

tacctgtgacactgcaccet $\mathrm{C} / \mathrm{T}$ acggccaccogactactttg

Fig. 1. Continued 
NADH ubiquinone oxidoreductase 1 alpha subcomplex 8 (NDUFA8): AL162423.10

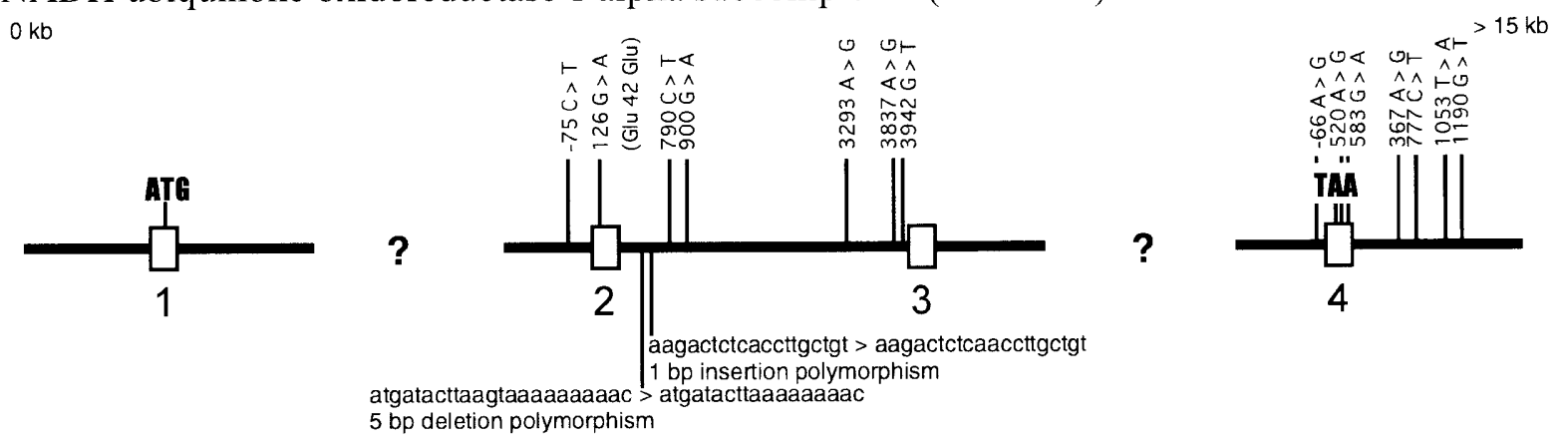

1 (intron 1 -75)

2 (coding region 126) dbSNP ID:rs4679

tttgtgttctctattctgac $\mathrm{C} / \mathrm{T}$ cgcatgaggtaagetgaga

3 (intron 2790 )

4 (intron 2 900)

5 (intron 2 3293) dbSNP ID:rs1537005

6 (intron 23837 )

7 (intron 2 3942)

8 (intron $3-66$ )

tgtgataagcccaacaagga G/A (Glu 42 Glu) tttatgctctgccgctggga caaacctagacaaagtgtgc $\mathrm{C} / \mathrm{T}$ ctttatccagaagtgagcag ttcaggagataaaaagctct $\mathbf{G} / \mathbf{A}$ attgctcaggcctgagatgg aactattcttttatacac $\mathbf{A} / \mathbf{G}$ gaagttaaatttctctagc gaagttgtcttgtaagtgag $A / G$ taagaatatgtactcacata tcattgttttgcaaagagat $G / T$ ccctaacccagctttctt gaggagacaccaggaggcgc A/G ttgatggttacagattcctc

9 ( 3 ' untranslated region 520) tt atttctggaccaagtaa $A / G$ gatgggtccgtggccacac

10 (3' untranslated region 583) dbSNP ID: rs6822

11 (3' flanking region 367)

12 ( 3 , flanking region 777 )

13 (3, flanking region 1053)

14 (3' flanking region 1190 )

actgatgaaaacgcccatgc G/A gtttgcatcgactgatagtg gtcatacaaggggagcctcC A/G ggatagaagtgcagaaact attctttttcactactagg $\mathrm{C} / \mathrm{T}$ tgtttcctccacatctgact aaagaaaagcactgtgtga $\mathbf{T} / \mathbf{A}$ ctgccatggcegcttctgca gattctctaatgaaaataa G/T acttttttgcatttttt

$n 8$ NADH ubiquinone oxidoreductase 1 alpha/beta subcomplex 1 (NDUFAB1): AC008870.6

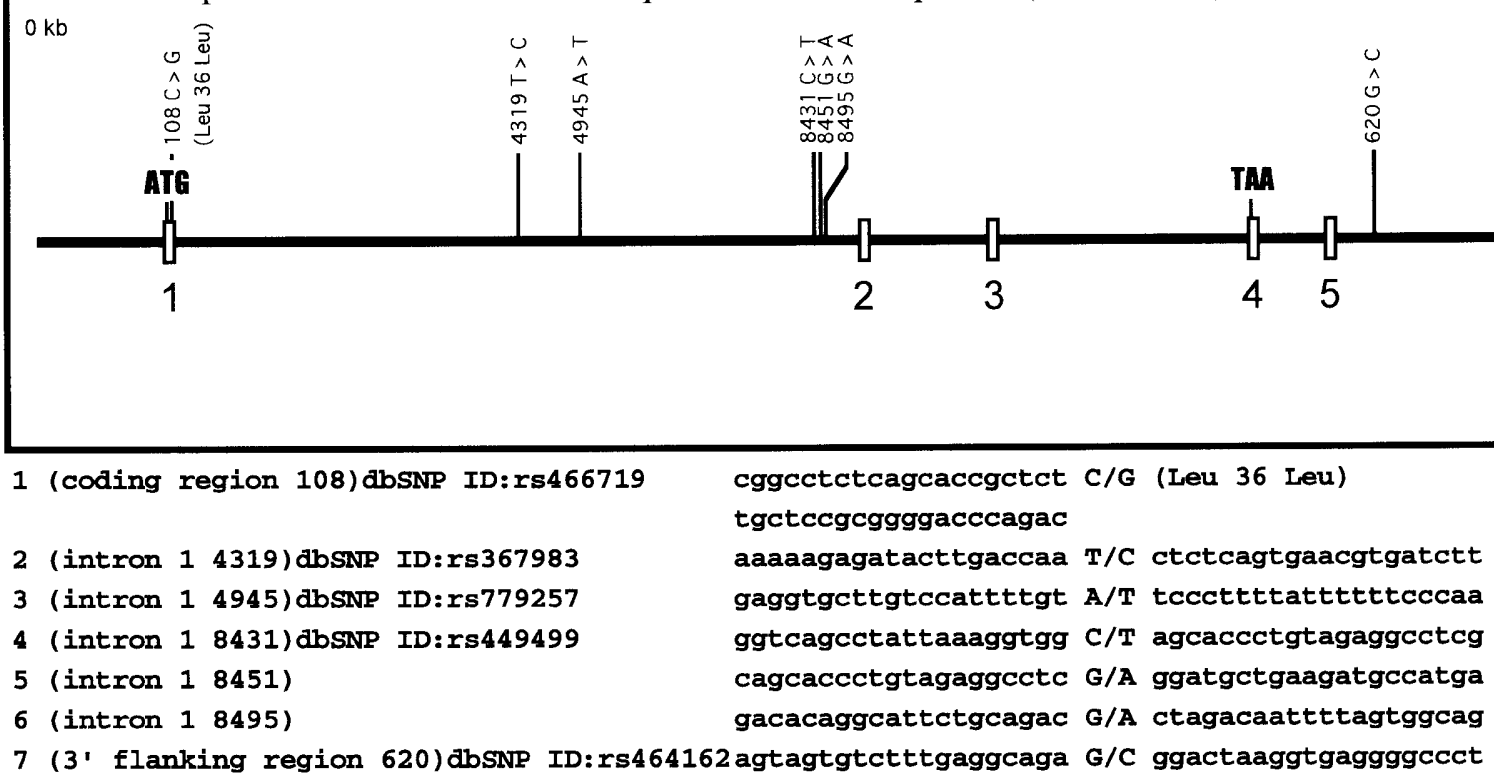

Fig. 1. Continued 
$n 9$ NADH ubiquinone oxidoreductase 1 alpha subcomplex 9 (NDUFA9): AC005832.1

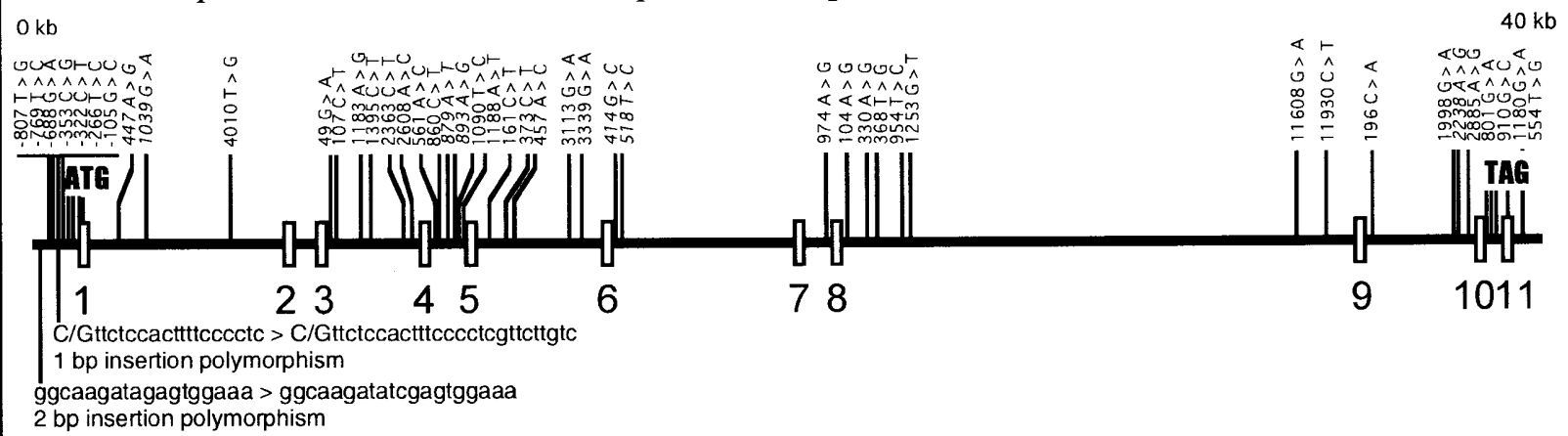

1 (5' flanking region -807)

2 (5' flanking region -769)

3 (5' flanking region -688) dbSNP ID:rs979878

4 (5' flanking region -353)

5 (5' flanking region -322)

6 (5' flanking region -266) dbSNP ID:rs979877

7 (5' untranslated region -105) dbSNP ID:rs714621

8 (intron 1 447)

9 (intron 1 1039)

10 (intron 1 4010)

11 (intron 3 49)

12 (intron 3 107)

13 (intron 3 1183)

14 (intron 3 1395)

15 (intron 3 2363)

16 (intron 3 2608)

17 (intron 4 561)

18 (intron 4 860)

19 (intron 4 879)

20 (intron 4 893)

21 (intron 4 1090)

22 (intron 4 1188)

23 (intron 5 161)

24 (intron 5 373)

25 (intron 5 457)

26 (intron 5 3113)

27 (intron 5 3339)

28 (intron 6 414)

29 (intron 6 518)

30 (intron 7 974)

31 (intron 8 104) dbSNP ID:rs1009633

32 (intron 8 330) dbSNP ID:rs1009634

33 (intron 8 368)

34 (intron 8 954)

35 (intron 8 1253)

36 (intron 811608 )

37 (intron 8 11930)

38 (intron 9 196) dbSNP ID:rs1029767

39 (intron 9 1998)

40 (intron 9 2238)

41 (intron 9 2885)

42 (intron 10 801)

43 (intron 10 910)

44 (intron 10 1180)

45 (3' flanking region 554) gatggctctttgtagaacaa T/G gcagattctcaaaggtgacc accacagttaaagaaaaaat $\mathrm{T} / \mathrm{C}$ acaagccattgcgctagaga cacacatggccactaaattt $\mathbf{G / A}$ cataatattcaggaagttc cacaccctattttggtttct $\mathrm{c} / \mathrm{G}$ ttctccacttteccctcgt ttcccetcgttcttgtcccc $\mathrm{C} / \mathrm{T}$ ctttctctctcctgggccc cggagggctacgcetccttc T/C tcctcoggactcaccagggc acaatctacccgaaaccgtc G/C tttcttggttagcgcttgcg attcatatgagcacaatgga $A / G$ atgataatattacaatacca ggcttgatgttcagcctgag $G / A$ caagaattaggagtgtttag aatgtatccaaaagagattc $\mathbf{T} / \mathbf{G}$ cattcctgccatatgaagaa gacaaatataaattactaag G/A tcattttaggagtgatagg aatttcttcccagaatggac $\mathrm{C} / \mathrm{T}$ aaaggcatcctctgttccca atctctggtaatattcatac $\mathbf{A} / \mathbf{G}$ gattattgtaatccetta attcctagttctttgtccct $\mathrm{C} / \mathrm{T}$ aggttgttggtcaccttgt agaaaatagtcatgaatggc C/T ccaactaacactagtcttta gtcatttgattacctgagta $\mathrm{A} / \mathrm{C}$ agtgtactgttacctgtttg attttataaattctttgatg $\mathrm{A} / \mathrm{C}$ cttgggggtcttatcaact attgtgtagagtaatgacag $\mathrm{C} / \mathrm{T}$ agagctgtcaactttttaa gcagagctgtcaacttttt $A / T$ aaaaataatttagcttaa tttttaaaaaataattt $A / G$ gcttaaaaaaattaaaat atcattgctgtttaaaagtt $\mathrm{T} / \mathrm{C}$ aagtagtgtgaatttcagta aaccaatcctttattttt $\mathrm{A} / \mathrm{T}$ tcttccagaaacttgatt gggtgtgtgtgatgttttga $\mathrm{C} / \mathrm{T}$ gtttgattgattgccttct cttctcacccetgcactg $\mathrm{C} / \mathrm{T}$ agtggtttgtgccactct gccagggaagatgcctattc $\mathrm{A} / \mathrm{C}$ cacagtgcttatgctcctt gatttttctccttctcaat G/A taagcttccettaaaataaa tctaaactcaaaacaggttt G/A tttggttattgtttaggctg tatagttttgcctttccag $G / C$ atattacatatatggttaga cttcattctttcatagc $T / C$ tgatagctcattctttata ggattatgcgtacttggaaa $\mathbf{A} / \mathbf{G}$ tacttggatagcggtgatta gttgcacttttacaatatca $\mathrm{A} / \mathrm{G}$ aatctcctcattgcttgcat acaaaacactttagggagt $\mathrm{A} / \mathrm{G}$ agtatggttataaaccaca acattaattttgatggagta $T / G$ cacaatgcctccagaggctg gcatgcaatcagttatatag $T / C$ ctagataagaattacaattc tcctcttgaaattgtagata G/T gtatctacacatttctcatc gaaaagatagatgtataaat G/A accaaaaattcgtgaagaaa ctacaaatatattctaaatg C/T gtaatcatggataagtacaa atattggaacattaaatctt $\mathbf{C} / \mathbf{A}$ ccaattccaaaagtgaatc tgtttttcaagcctttaaac G/A gctgtggaaccctgtgctca ccagctacttgggaggctga A/G gtgggaggatcacttgagcc acagcggtctgtcttcctgc $\mathbf{A} / \mathbf{G}$ gttctcataggctagcttac tacactaaagtgtctcttac G/A tttatacttgagaaagtgtt tgcagactttcaggtgggta G/C gatgagggattgctgctgct aaaactgagtcagaacgccC G/A tgctcagaaaacaggggcgt gtgccagcacttaggaatta $T / G$ gaccttctaatgaagttctt

Fig. 1. Continued 
$n 10$ NADH ubiquinone oxidoreductase Fe-S protein 1 (NDUFS1): AC007383.4

$\begin{array}{llll}0 & \end{array}$
$n 1 1 \longdiv { \mathrm { O } _ { \mathrm { Kb } } }$ (ADH ubiquinone oxidoreductase Fe-S protein 3 (NDUFS3): AC067943.4
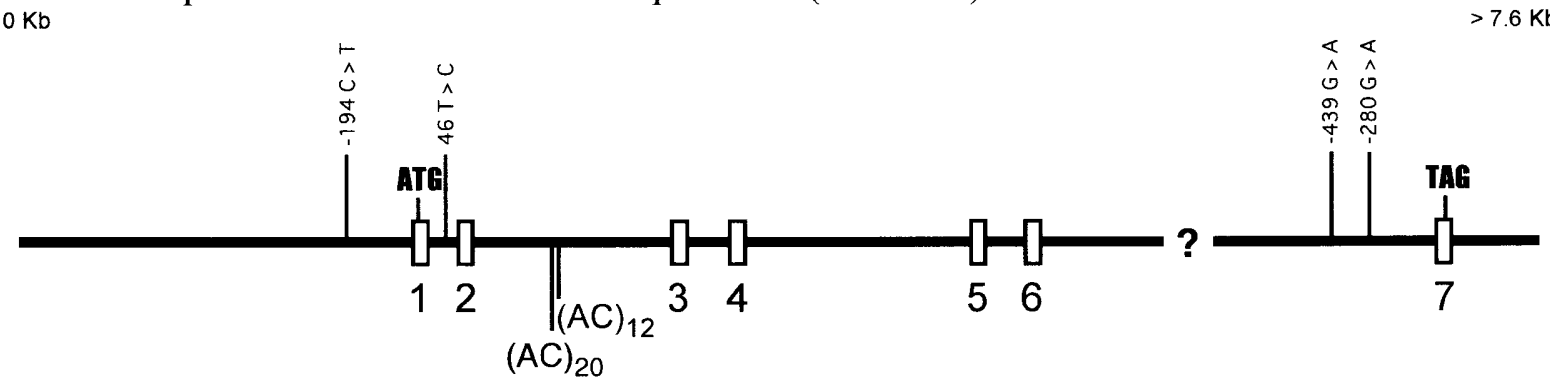

1 (5' flanking region -194)

2 (intron 146 )

3 (intron $6-439)$

4 (intron $6-280$ )

tctgccacaaggagctagga $\mathrm{C} / \mathrm{T}$ cacgctcacctcacgatttc cggggtcaggcgcagcggcg $\mathrm{T} / \mathrm{C}$ gcccagtgcagagagctcct aaagctgtgtcaaatgtact G/A ctttagatctggactgtgaa ggtgggtgagcagtcagttc G/A gagctcctgatgtgggagtg

Fig. 1. Continued 
$n 12$ NADH ubiquinone oxidoreductase Fe-S protein 4 (NDUFS4): AC024569.3

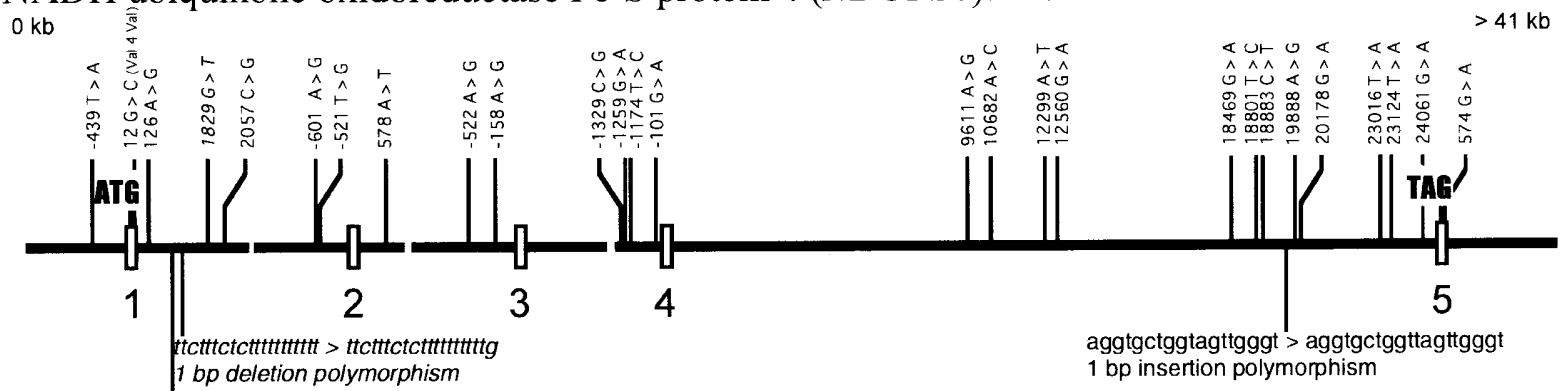

tgattttttttttttggc > tgatttttttttttggc

$1 \mathrm{bp}$ deletion polymorphism

1 (5, flanking region -439 )

2 (coding region 12) dbSNP ID: $5 \$ 1127795$

3 (intron 1 126) dbSNP ID: rs 1532163

4 (intron 1 1829)

5 (intron 1 2057)

6 (intron 1 -601) dbSNP ID: 83365358

7 (intron 1 -521)

8 (intron 2 578) dbSNP ID: rs409313

8 (intron 2 578) dbSNP ID:r8409313

9 (intron $2-522$ ) dbSNP ID: $r 8810858$
10 (intron 2 -158) dbSNP ID: $r 831305$

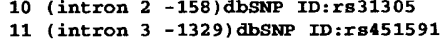

11 (intron $3-1329$ )

12 (intron $3-1259$ )

14 (intron 3 -101) dbSNP ID: rs 1490769

15 (intron 4 9611) dbSNP ID: rs189849

16 (intron 4 10682)

17 (intron 12299 )

17 (intron 412299 )

18 (intron 12560 )

19 (intron 418469 )

20 (intron 4 18801)

21 (intron 4 18883)

22 (intron 4 19888)

24 (intron 423016 )

25 (intron 43124 )

26 (intron 4 24061) dbSNP ID: rs439552

27 ( 3 ' untranslated region 574) dbSNP ID:rs567 aactgaatacagcctgtcc $T / \mathbf{A}$ gagggettgcaaagtgaatc

tgcagcaagatggcggcggt $\mathrm{G} / \mathrm{C}$ (Val 4 Val) tcaatgtcagtggtactgag

ttgaccetttetcgggaga $\mathrm{A} / \mathrm{G}$ gtcgggcggactagggactg

gaaaaaaatct taatgcca $G / T$ ggaagacgttttttaatac

at taatgggaaatctacat C/G taaabtcattttattgtaa

taagtaattggagtgagagt $\mathrm{A} / \mathrm{G}$ ggaaatgctaata taaagg

ttcattt taactaattttat T/G tctcccattttgtgaatggg

geagcatcactataaan $A / T$ ttttagagtgtogtctgtt

gcagccatcacteataaat $A / G$ tttagagtgtggtctgtta traaagttgttaaatthaca $\mathbf{A} / G$ ctttacaaaaggteagg gtatctaaatagtaatttac A/G ttgtaaagtatcagaatggt ttctgttctcagtatatata $\mathrm{C} / \mathrm{G}$ tttaactatcaactggaaa ataaat tatgatattatta G/A tactaatatagccagccata aatatataattataggaa $\mathrm{T} / \mathrm{C}$ ctcagagtagcaaccatggt taaatattccatcaataat $\mathbf{G / A}$ tact tatt gattt tatagt tcaaatacaatt tgttatca $\mathrm{A} / \mathrm{G}$ ttgtatt tgagatacagttg cacaatataggcacaaact $\mathrm{A} / \mathrm{C}$ ctaccaa agcactaacaagt tttactatatagatatatg $\mathrm{A} / \mathrm{T}$ atagactatagagtatctet accaatagotattatgca G/A gctcatctttttatataag tgtgttctaatttctaatg G/A ctgettttcagatatactt ge ggaaactectetgcan T/A gtatccgaaacctotgttat cattattctgaaccatteac c/T gectactgtacccacccc tcgcacagctgagaagagca $\mathrm{A} / \mathrm{G}$ ggggctggttttcagtacc agaaagatgagtataattC $G / \mathbf{A}$ tctaacttacccattcttaa ctactctgtgaaagtaaggt $\mathbf{T} / \mathbf{A}$ atgttgaacaagtaaattaa actttctttggagatggagt $\mathbf{T} / \mathbf{A}$ ccagcagttgggaatgtaat atacgtttacatattttect $\mathrm{G} / \mathbf{A}$ tatccettrggaagagttc gactgtgaataaagtcagct G/A tgcagtatttatagtccatg

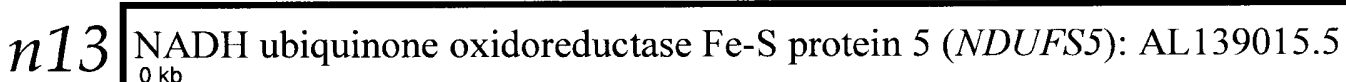

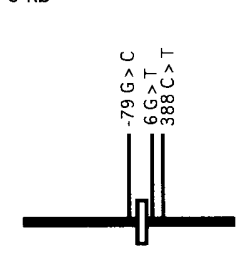

1

\section{?}

taaacaaagatgtgtggtct > taaacaaaggtgtggtct 2 bp deletion polymorphism

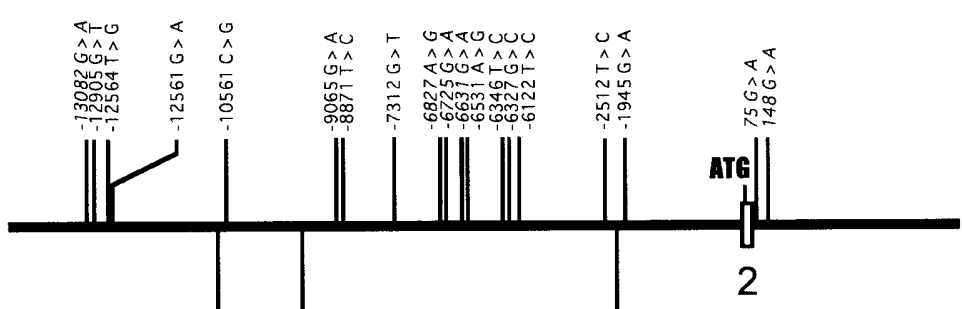

ttccctttacttgttttcc $>$ ttccctttattgttttcc

1 bp deletion polymorphism

actaccctgaaaaaagaag > actaccctgaaaaagaag

1 bp deletion polymorphism

1 (5' flanking region -79) dbSNP ID:rs1122471

2 (intron 16 ) dbSNP ID: $x \mathbf{1 1 2 2 4 7 2}$

3 (intron 1388 )

4 (intron 1 -13082)

5 (intron 1 -12905)

6 (intron $1-12564$ )

7 (intron $1-12561$ )

8 (intron $1-10561$ )

9 (intron 1 -9065)

10 (intron $1-8871$ )

11 (intron 1 -7312)

12 (intron $1-6827$ )

13 (intron $1-6725$ )

14 (intron $1-6631$ )

15 (intron $1-6531$ )

16 (intron $1-6346$ )

17 (intron $1-6327$ )

18 (intron $1-6122$ )

19 (intron 1 -2512)

20 (intron 1 -1945)

21 (intron 2 75)

22 (intron 2 148)

23 (3' flanking region 150) ggctcggtttctatggtttt $\mathrm{G} / \mathrm{C}$ tggggagacggctgagggaa acgagcatcgggtaggtagg $\mathbf{G} / \mathbf{T}$ gctgcttatgtccgcgcggc ccaaacatagccagcacttc C/T ggctgtaactccgggctgtt agtgagccgagattgcacca G/A tgcattccagcctgggcaac gttttcaacaaaggactcca $\mathrm{G} / \mathrm{T}$ agtagtagagaagtttctgt attttcatcacacctcaact $T / G$ aaggtataacagccttaaga ttcatcacacctcaacttaa G/A gtataacagccttaagaatg aacaatgtggtatagtgggg C/G gggtggtgagcaggtgtcat cctgatgctcctggctccag G/A gtagacctttccctttaga tcaccacgtgtctgtagata $\mathbf{T} / \mathrm{C}$ aggaccgcagaccttcgctt aatccttggcttctagaat $\mathbf{G} / \mathrm{T}$ ggtcactgatggtatataat aacctctgcctcccogattc A/G cgccattctcctgcctcagc agtagagacggggtttcacc $\mathrm{G} / \mathrm{A}$ tgttagccagcatggtctcg aggcgtgagccactgcgccC G/A gcctagaccttcttcttat cccaacagctcccaatgtaa $A / G$ acagatctattaatattctg gcaacagatcttgacctata $T / C$ ccatagggtacagctgagg atcccatagggtacagctga G/C gactttaatcagaaaaggag tagccttgcttttactctac T/C gttcctcccaaatcacacc acaaactcttaatgcgaatt $T / C$ tgcagatcaaagtgggctta tttaatctccttaaatttc G/A caattcacaacctagggta tttttttttttttgagac $G / A$ aagtctcactct tgtcccct ctgtagcctctgcctcccag G/A ttcaggcgattcgcgtacct cagattcaagtggttctcct $G / C$ cctcagcctcccaagtagct

Fig. 1. Continued 


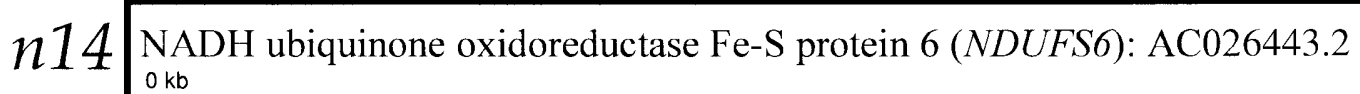

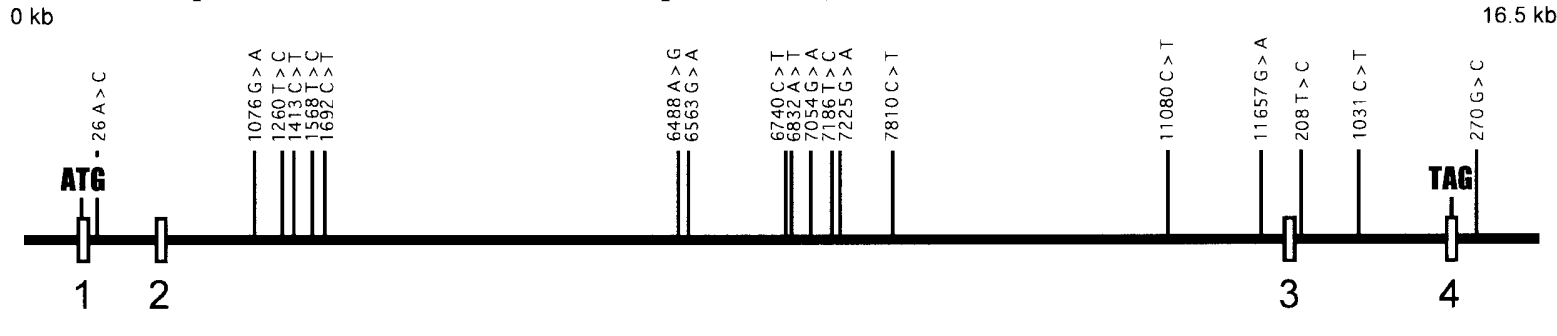

1 (intron 126 )

2 (intron 2 1076)

3 (intron 2 1260)

4 (intron 2 1413)

5 (intron 2 1568)

6 (intron 2 1692)

7 (intron 26488 )

8 (intron 26563 )

9 (intron 2 6740)

10 (intron 2 6832)

11 (intron 2 7054)

12 (intron 2 7186)

13 (intron 2 7225)

14 (intron 2 7810)

15 (intron 2 11080)

16 (intron 211657 )

17 (intron 3 208)

18 (intron 3 1031)

19 (3' flanking region 270 ) ggcegctgggtacaggatgc A/C cettcetcagcegcactc ggatcatggtggtggagagg $\mathbf{G / A}$ gcttgtgtctggtgggtttg cagttgtcgagtaagtggtg $T / C$ atagggtaagtgetctttct caargagctcatggcattg $\mathrm{C} / \mathrm{T}$ gaatgggacatttcttccgt tggagaaggggaggtttctc $T / C$ tagtgtggatgcggtatggt gaccgtggtgacggaggttt $\mathrm{C} / \mathrm{T}$ ctgggcatcgatgggtggtt tagcttaaataattattggC A/G ttcatgttcagaatgcctga tttaactttatttaaat $G / A$ tccatgaatggggtcggtat aagatt aaacctacatat $\mathrm{C} / \mathrm{T}$ tttatgcccaatcattgat gcgagggactcattttacag $\mathrm{A} / \mathrm{T}$ ggttggacacttcactgtgt ttcactgccggagcttggcc G/A tgtgaacccggagccgggct ggtcagggtcacccttgagc T/C gcgcacactaaatgacggga gagggcatcccgcgtcagtc G/A ccagtgtcgaggcgtcagca cttccactctggggcgggga $C / T$ gctgtagaaggagcacaaag gtaactgttcagtgctttct $\mathrm{C} / \mathrm{T}$ ctttggatttcatgtaatc gggacagaacgatgtggtgg G/A gagaagagggcgtggcagag cgaaaaccccttcaactg T/C gaagtggtgggcggcatgtt ctagagtgggactgggcace C/T ggcatgtcectcctggget gcttcagagagccaaggtgg $\mathrm{G} / \mathrm{C}$ tcttgaggtgcatagtgaag $n 1 5 \longdiv { \substack { \text { NADH ubiquinone oxidoreductase Fe-S protein } 8 \text { (NDUFS8): AC034259.2 } \\ \text { kb } } }$

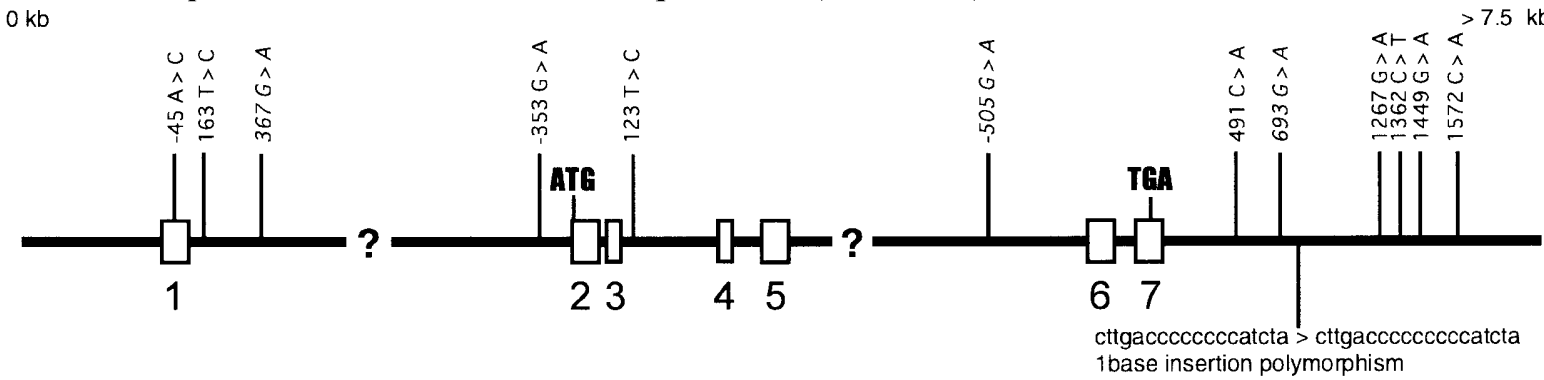

1 (5' untranslated region -45 )

2 (intron 1 163)

3 (intron 1 367) dbSNP ID:rs1122731

4 (intron 1 -353) dbSNP ID: rs999571

5 (intron 3 123)

6 (intron $6-505)$

7 (3' flanking region 491)

8 (3' flanking region 693)

9 (3' flanking region 1267)

10 (3' flanking region 1362)

11 (3' flanking region 1449)

12 (3' flanking region 1572) agtgtagcctccgcetcccg A/C ttgactggectgcttggcaa aggtgcagcggggagccggc T/C ctcagggcgcatgcgccgcc ctctggggtgcccttggcat $\mathrm{G} / \mathrm{A}$ tacgggctcccctaattccc cgctctcagtgcatgctggc G/A ggggctggtaatgttgtttt tctctgagcctgtttccact $T / C$ ttaaaatgattatggtgatg aggcaaggcaggccgggcac G/A gtggctcacgcttgtaatcc ggccetgagctggcctgcgt $\mathrm{C} / \mathrm{A}$ cagccacatcctctttcctg ttcacttcatttgcagtgag $\mathbf{G / A}$ aaaccagctccgagaggtga ttttcccagacgtaaccgcc G/A tcagagcgtggcatggagcc cgctgggttctttccettac C/T gtggtctccaggcacttac tgtcagaacaggcctatggc G/A cccaaccacaagtccccaa cagccccacaggcetgtgct $\mathrm{C} / \mathrm{A}$ gctgtgtggggcttagggat

Fig. 1. Continued 


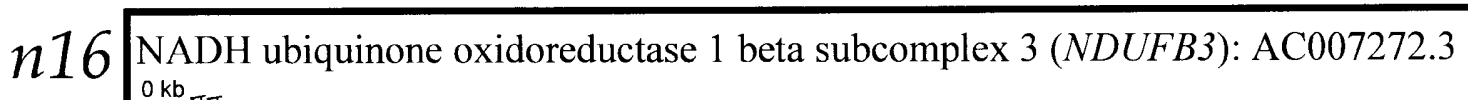

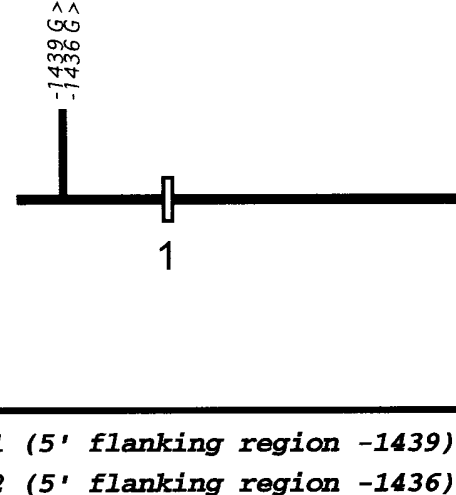

ttaaaagtgactttttct G/A CC G/A ggcacggtggctcacgcctg

$n 17$ NADH ubiquinone oxidoreductase 1 beta subcomplex 5 (NDUFB5): AC068361.2
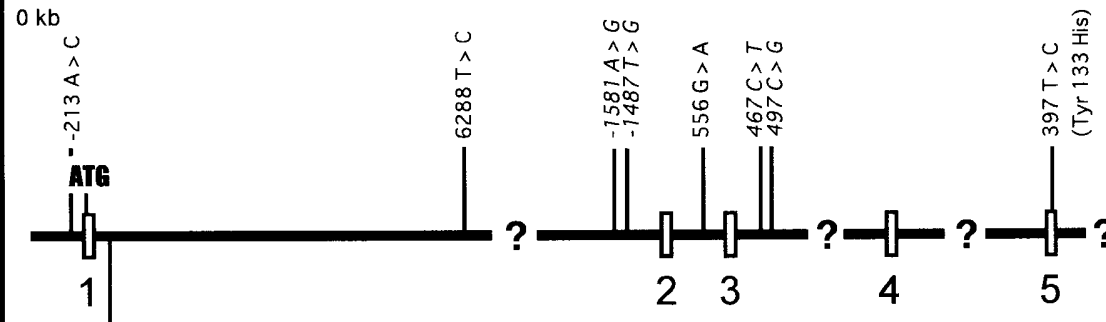

taaaacgttgttattcaccat > taaaacgttattcacca 3 base deletion polymorphism

1 (5' flanking region -213)

2 (intron 1 6288)

3 (intron 1 -1581)

4 (intron 1 -1487)

5 (intron 2556 )

6 (intron 3 467)

7 (intron 3 497)

8 (coding region 397) ggcggatgaaactctcctac $\mathrm{A} / \mathrm{C}$ aagaagggccaaaccggccg ggggatgttgattacctagg $\mathrm{T} / \mathrm{C}$ cagtaaagtaaagaaggcat cttctgggccactgtatcct $\mathrm{A} / \mathrm{G}$ tttctttccettgttaccct ccctcttagaccgtatatag $T / G$ tctagcataggatctgcaca ttgtctggaccatctgccac G/A gtagataaagctctgaatca ggcgccatcgcactccagcc $C / T$ gggcaacagagtgagactct agtgagactctgtccccccc $C / G$ caaaaaaaactataatcct atgatagtcctgaaaagata T/C (TYx $133 \mathrm{His)} \mathrm{atgaaagaacaatggccgtc}$

Fig. 1. Continued 

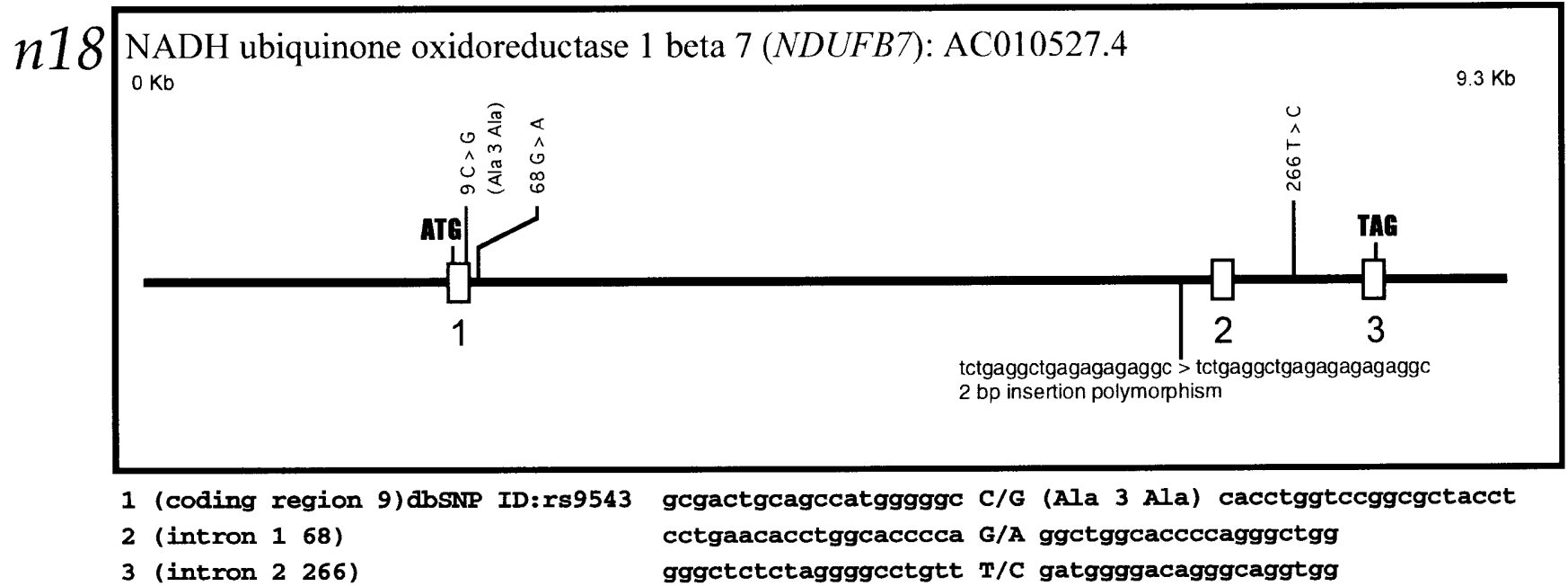

Fig. 1. Continued

NADH ubiquinone oxidoreductase family

Respiratory NADH ubiquinone oxidoreductases (also known as complex I, which is embedded in the inner mitochondrial membrane; EC1.6.5.3) serve to dehydrogenate NADH and to shuttle electrons to coenzyme Q. This electron transport generates a proton gradient across the inner mitochondrial membrane, to provide the proton-motive force for ATP synthesis (Guenebaut et al. 1998). The total human complex consists of 42 or more subunits, seven of which are encoded by the mitochondrial genome and the remainder by the nuclear genome. So far, complete gene structures have been reported for six of the 35 nuclear members of this family, and chromosomal localization is known for 26 of the 35 (for reviews see Ton et al. 1997; Loeffen et al. 1998; Smeitink and van den Heuvel 1999). We analyzed 18 complex I genes by direct sequencing, based on their cDNA sequences (Fig. 1. $\mathrm{n} 1-\mathrm{n} 18)$. A total of $241 \mathrm{SNPs}$ were identified from the 18 loci: 11 in coding elements, 22 in $5^{\prime}$ flanking regions, four in a $5^{\prime}$ untranslated region, 170 in introns, five in $3^{\prime}$ untranslated regions, and 29 in $3^{\prime}$ flanking regions. Of these, $187(78 \%)$ were novel and 38 were located within regions corresponding to repetitive sequences predicted by the RepeatMasker program. Of the 11 SNPs identified within coding elements, four (Leu81Val in the NDUFA3 gene, Ala9Val in the NDUFA6 gene, Pro66Ala in the NDUFA7 gene, and Tyr133His in the NDUFB5 gene) were nonsynonymous and had not been reported previously. With regard to distribution, we found 11 SNPs within the 4.4-kb NDUFA2 genomic locus, while only two were detected in the $18-\mathrm{kb}$ genomic sequence containing the NDUFB3 gene.

The SNP collection reported here provides genetic data that should be helpful for personalized medical service and also for identifying genes associated with drug efficacy and/ or side effects. We believe that, in the near future, using an SNP-based approach to predict individual differences in drug efficacy or toxicity on the basis of genetic factors will be a realistic approach to treatment of human diseases or toxic conditions. Finally, we hope that the virtual experiments made possible by our catalog will accelerate certain aspects of human genetic research.

\section{References}

Ashmarin IP, Danilova RA, Obukhova MF, Moskvitina TA, Prosorovsky VN (2000) Main ethanol metabolizing alcohol dehydrogenases (ADH I and ADH IV): biochemical functions and physiological manifestations. FEBS Lett 486:49-51

Duester G, Farres J, Felder MR, Holmes RS, Hoog JO, Pares X, Plapp BV, Yin SJ, Jornvall H (1999) Recommended nomenclature for the vertebrate alcohol dehydrogenase gene family. Biochem Pharmacol 5:389-395

Guenebaut V, Schlitt A, Weiss H, Leonard K, Friedrich T (1998) Consistent structure between bacterial and mitochondrial NADH:ubiquinone oxidoreductase (complex I). J Mol Biol 276:105112

Halushka MK, Fan JB, Bentley K, Hsie L, Shen N, Weder A, Cooper R, Lipshutz R, Chakravarti A (1999) Patterns of single-nucleotide polymorphisms in candidate genes for blood-pressure homeostasis. Nat Genet 22:239-247

Hayes JD, Pulford DJ (1995) The glutathione S-transferase supergene family: regulation of GST and the contribution of the isoenzymes to cancer chemoprotection and drug resistance. Crit Rev Biochem Mol Bio 30:445-600

Iida A, Sekine A, Saito A, Kitamura Y, Kitamoto T, Osawa S, Mishima C, Nakamura Y (2001) Catalog of 320 single nucleotide polymorphisms (SNPs) in 20 quinone oxidoreductase and sulfotransferase genes. J Hum Genet 46:225-240

Kruglyak L (1997) The use of a genetic map of biallelic markers in linkage studies. Nat Genet 17:21-24

Loeffen JL, Triepels RH, van den Heuvel LP, Schuelke M, Buskens CA, Smeets RJ, Trijbels JM, Smeitink JA (1998) cDNA of eight nuclear encoded subunits of NADH:ubiquinone oxidoreductase: human complex I cDNA characterization completed. Biochem Biophys Res Commun 253:415-422

McCarthy JJ, Hilfiker R (2000) The use of single-nucleotide polymorphism maps in pharmacogenomics. Nat Biotechnol 18:505-508

Nickerson DA, Tobe VO, Taylor SL (1997) PolyPhred: automating the detection and genotyping of single nucleotide substitutions using fluorescence-based resequencing. Nucleic Acids Res 25:2745-2751

Ohnishi Y, Tanaka T, Yamada R, Suematsu K, Minami M, Fujii K, Hoki N, Kodama K, Nagata S, Hayashi T, Kinoshita N, Sato H, Sato 
H, Kuzuya T, Takeda H, Hori M, Nakamura Y (2000) Identification of 187 single nucleotide polymorphisms (SNPs) among 41 candidate genes for ischemic heart disease in the Japanese population. Hum Genet 106:288-292

Risch N, Merikangas K (1996) The future of genetic studies of complex human diseases. Science 273:1516-1517

Saito S, Iida A, Sekine A, Eguchi C, Miura Y, Nakamura Y (2001) Seventy genetic variations in human microsomal and soluble epoxide hydrolase (EPHX1 and EPHX2) genes in the Japanese population. J Hum Genet 46:325-329

Sekine A, Saito S, Iida A, Mitsunobu Y, Higuchi S, Harigae S, Nakamura Y (2001) Identification of single nucleotide polymorphisms (SNPs) of human N-acetyltransferase genes NAT1, NAT2, AANAT, ARD1 and L1CAM in the Japanese population. J Hum Genet 46:314-319

Sherry ST, Ward M, Sirotkin K (2000) Use of molecular variation in the NCBI dbSNP database. Hum Mut 15:68-75
Sherry ST, Ward MH, Kholodov M, Baker J, Phan L, Smigielski EM, Sirotkin K (2001) dbSNP: the NCBI database of genetic variation. Nucleic Acids Res 29:308-311

Smeitink J, van den Heuvel L (1999) Human mitochondrial complex I in health and disease. Am J Hum Genet 64:1505-1510

Strange RC, Jones PW, Fryer AA (2000) Glutathione S-transferase: genetics and role in toxicology. Toxicol Lett 112-113:357-363

Ton C, Hwang DM, Dempsey AA, Liew CC (1997) Identification and primary structure of five human $\mathrm{NADH}$-ubiquinone oxidoreductase subunits. Biochem Biophys Res Commun 241:589-594

Wang DG, Fan JB, Siao CJ, Berno A, Young P, Sapolsky R, Ghandour G, Perkins N, Winchester E, Spencer J, Kruglyak L, Stein L, Hsie L, Topaloglou T, Hubbell E, Robinson E, Mittmann M, Morris MS, Shen N, Kilburn D, Rioux J, Nusbaum C, Rozen S, Hudson TJ, Lander ES (1998) Large-scale identification, mapping, and genotyping of single-nucleotide polymorphisms in the human genome. Science 280:1077-1082 